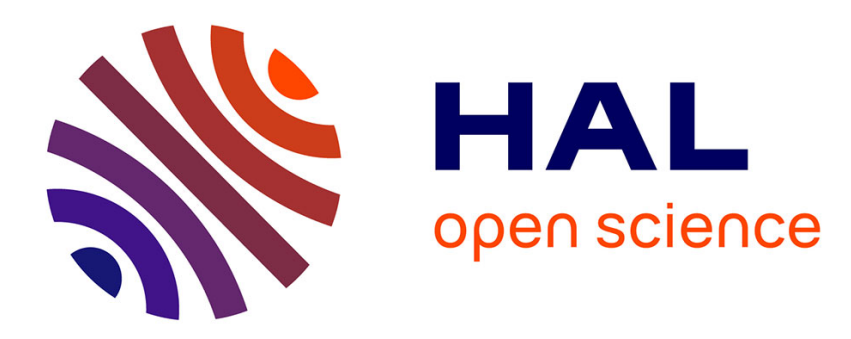

\title{
Nonlocal impact ionization and avalanche multiplication
} G J Rees, J P R David

\section{To cite this version:}

G J Rees, J P R David. Nonlocal impact ionization and avalanche multiplication. Journal of Physics D: Applied Physics, 2010, 43 (24), pp.243001. 10.1088/0022-3727/43/24/243001 . hal-00569627

\section{HAL Id: hal-00569627 https://hal.science/hal-00569627}

Submitted on 25 Feb 2011

HAL is a multi-disciplinary open access archive for the deposit and dissemination of scientific research documents, whether they are published or not. The documents may come from teaching and research institutions in France or abroad, or from public or private research centers.
L'archive ouverte pluridisciplinaire HAL, est destinée au dépôt et à la diffusion de documents scientifiques de niveau recherche, publiés ou non, émanant des établissements d'enseignement et de recherche français ou étrangers, des laboratoires publics ou privés. 


\title{
Nonlocal impact ionization and avalanche multiplication
}

\author{
G J Rees and J P R David \\ Department of Electronic and Electrical Engineering \\ Sheffield University \\ Sheffield S1 3JD \\ In memory of Peter Robson, who inspired and \\ encouraged scientists and engineers, young and old
}

\begin{abstract}
Impact ionization and avalanche multiplication are conventionally described in terms of ionization coefficients which depend only upon the local electric field. Such a description takes no account of the effect of ionization dead space, within which the population distribution, and hence the ionization coefficient of carriers injected cool approach equilibrium with the high electric field, inhibiting ionization and reducing multiplication. This effect, which increases in importance as device dimensions are reduced, clearly benefits such high field devices as transistors by suppressing parasitic avalanche multiplication. It also improves the performance of avalanche photodiodes (APDs) by reducing the spatial randomness of impact ionization, so that the resulting excess multiplication noise is also reduced. It reduces temperature sensitivity and may also further enhance APD speed. This article reviews these effects and some theoretical models used to describe them.
\end{abstract}

\section{Introduction}

At the highest electric fields all semiconductors break down ${ }^{1}$. Free carriers may be generated by tunnelling across the spatially tilted band gap, by optical excitation or by thermal emission from deep traps. They accelerate in the field to such high energies that, in falling back towards the band edge, they can release enough energy and crystal momentum to generate a further electron-hole pair in the process of impact ionization. Driven in opposite directions by the electric field these extra electrons and holes may give rise to a chain of ionization events, leading to charge multiplication. If the multiplication region is long enough and the field is high enough then the 
multiplication can grow without limit and an avalanche of current is generated which increases indefinitely until other effects supervene*.

Avalanche multiplication is parasitic in a variety of transistors, compromising their performance. More usefully it is exploited in Zener Diodes, used widely in electronic circuits to regulate voltage. It also provides the internal gain in an APD. These devices were originally operated in analogue mode, below breakdown, where the multiplication is kept finite and amplifies the photocurrent arising from weak optical signals. APDs are now also increasingly operated in Geiger mode, above breakdown, where the multiplication is high enough to permit detection of individual photons. The avalanche is then quenched, either by the drop in diode voltage provided by a series load resistor, or using more sophisticated circuitry.

The local ionization coefficients, $\alpha$ for electrons and $\beta$ for holes, conventionally used to describe avalanche multiplication, represent a carrier's mean rate of ionization per unit distance and are also equal to the inverse of the mean distance a carrier travels before ionizing. They increase rapidly with electric field, since only at high fields can carriers acquire enough energy to impact ionize and conserve both energy and crystal momentum in the process.

The term 'local' here means that the ionization coefficients depend only on the local electric field. This is never strictly true. Carriers injected 'cold' (at thermal energies, close to the band edge) must travel some distance down the field before their distribution in energy heats and their ionization coefficient reaches equilibrium with the field. Within this 'dead space' ionization is suppressed.

Early Si APDs were designed for operation with GaAs emitters, at a wavelength of around $800 \mathrm{~nm}$, for which the absorption length in $\mathrm{Si}$ is around $10 \mu \mathrm{m}$. Their multiplication region widths, $w$ were also made not much smaller, easily accomplished by the technology at that time. Consequently an applied bias of around $100 \mathrm{~V}$ was required to reduce the ionisation path lengths to comparable dimensions

\footnotetext{
* In narrow gap materials breakdown may be dominated by interband tunnelling.
} 
and achieve a multiplication of $\langle M\rangle \sim 100$. The dead space, of order a few tenths of a micron, could then be ignored and the local approximation was good.

InP based APDs are now used extensively to detect the longer wavelengths preferred for low loss in optical fibre communications systems. InP allows epitaxial growth of a variety of lattice matched, or controllably strained III-V alloys, including InGaAsP and InAlAs, and in the resulting heterostructure devices the multiplication and optical absorption regions can be grown from separate materials. Optical absorption in these materials is stronger because of their direct band gaps, so that shorter absorption regions can be employed. Moreover, the separate multiplication region can be made thin to increase device speed using modern growth techniques.

While the convenient local assumption is often made in the analysis and design of InP based APDs it is not always justified because the ionization dead space can constitute a significant fraction of the multiplication region. More recently, other materials systems have been proposed for use in APDs under conditions where the local approximation breaks down, including $\mathrm{SiC}$ devices used for visible blind UV detection $^{2}, \mathrm{Si} / \mathrm{SiGe}$ strained layer multiple heterostructures ${ }^{3}$ and impact ionization engineered structures ${ }^{4}$, designed to exploit the benefits which we shall see nonlocal impact ionization confers. Moreover, the submicron high field regions of modern transistors are all too short for the parasitic avalanche process to be treated locally.

In this paper we review understanding of impact ionization and multiplication when the local assumption is not justified, contrasting and assessing the predictions of the local and nonlocal models. While we shall focus mostly on the consequences for APDs we shall try to emphasise the underlying physics so that our comments may have more general application.

\section{Avalanche multiplication}

The terminology and analysis of multiplication in the local model follows the physics of the gas discharge process ${ }^{5}$ and is described widely in the literature ${ }^{6,7}$. We consider a multiplication region subject to a high electric field, such as the i-region of an idealised, reverse biased pin diode, which extends from $x=0$ to $x=w$ and in which electrons drift in the positive $x$-direction. The $\mathrm{p}^{+}$-type cap sits on top at $x=0$ and the 
$\mathrm{n}^{+}$-type substrate extends beyond $x=w$. A primary carrier pair injected at $x$ will, on average enjoy a multiplication $\langle M(x)\rangle$, meaning that an average number $\langle M(x)\rangle$ of carrier pairs (including the original pair) will be collected at the terminals. This quantity obeys the integral equation ${ }^{8}$ $<M(x)>=1+\int_{0}^{x} d x^{\prime} \beta\left(x^{\prime}\right)<M\left(x^{\prime}\right)>+\int_{x}^{w} d x^{\prime} \alpha\left(x^{\prime}\right)<M\left(x^{\prime}\right)>$. This equation states merely that the mean multiplication associated with carrier pair injection at $x$ results from multiplication chains generated in the interval $(0, x)$ from ionization by the primary hole, contributing the second term on the right hand side (RHS), from chains generated in the interval $(x, w)$ from ionization by the primary electron, contributing the third term, and from the primary pair, contributing unity.

Such recurrence relationships, in which the unknown quantity on the left appears again under an integral on the right, turn up repeatedly in the theory of avalanche multiplication, a process which is self regenerating. Differentiating this integral equation with respect to $x$ yields a differential equation for $\langle M(x)\rangle$ which may be solved, using the boundary relations provided by the integral equation at $x=0$ and $x=$ $w$, to yield ${ }^{8}$

$$
<M(x)>=\frac{\exp \left(-\int_{0}^{x} d x^{\prime} \gamma\left(x^{\prime}\right)\right)}{\left(1-\int_{0}^{w} d x^{\prime} \alpha\left(x^{\prime}\right) \exp \left(-\int_{0}^{x^{\prime}} d x^{\prime \prime} \gamma\left(x^{\prime \prime}\right)\right)\right)},
$$

where $\gamma(x) \equiv \alpha(x)-\beta(x)$.

In this treatment any position dependence of the ionization coefficients, $\alpha(x)$ and $\beta(x)$ in eqn. (1) arises because of that of the electric field, which $\alpha$ and $\beta$ are assumed to track faithfully, and not from nonlocal effects. When the field, and hence $\alpha$ and $\beta$, are independent of position then eqn. (1) simplifies to

$$
<M(x)>=\frac{\gamma \exp (-\gamma x)}{\alpha \exp (-\gamma w)-\beta} .
$$

In particular, when carriers are injected at $x=0$ the holes are immediately collected in the $\mathrm{p}^{+}$-region and only the electrons go on to ionize, giving a (mean) multiplication 


$$
M_{e} \equiv<M(0)>=\frac{\alpha-\beta}{\alpha \exp \{(\beta-\alpha) w\}-\beta}
$$

for pure electron injection. Similarly, for pure hole injection at $x=w$

$$
M_{h} \equiv<M(w)>=\frac{\alpha-\beta}{\alpha-\beta \exp \{(\alpha-\beta) w\}} .
$$

Avalanche breakdown, corresponding to a divergence in multiplication, occurs when the denominators of the right hand sides of these equations approach zero in the increasing electric field, because of the positive feedback provided by successive impact ionization by daughter electrons and holes.

Eqns. (3) may be solved for $\alpha$ and $\beta$ to find

$$
\alpha=\frac{1}{w}\left(\frac{M_{e}-1}{M_{e}-M_{h}}\right) \ln \left(\frac{M_{e}}{M_{h}}\right)
$$

and

$$
\beta=\frac{1}{w}\left(\frac{M_{h}-1}{M_{h}-M_{e}}\right) \ln \left(\frac{M_{h}}{M_{e}}\right),
$$

from which we can deduce the field dependence of ionization coefficients from measurements of multiplication made under conditions of pure electron and pure hole injection. From measurements on a range of devices with different multiplication region widths we can extend the field range investigated, since narrower devices will need larger ionization coefficients, and so higher fields, to maintain multiplication. Figure (1) shows $\alpha$ deduced in this way from measurements ${ }^{9}$ of $M_{e}$ and $M_{h}$ on $\mathrm{Al}_{0.6} \mathrm{Ga}_{0.4} \mathrm{As} \mathrm{p}-\mathrm{i}-\mathrm{n}$ diodes (note the logarithmic scale for $\alpha$ and the inverse scale for the field).

Evidently the ionization coefficient increases strongly with electric field and the values of $\alpha$ deduced from measurements on devices of different multiplication region width all converge to an envelope at high fields. However, at the low end of the field range for each device width the ionization coefficient droops below the envelope and this low field droop sets in at fields which increase as the devices narrow. This effect is well known to be a consequence of ionization dead space. The energy distribution of charge carriers approaches an equilibrium in a uniform electric field and so the ionization coefficient rises to a stable, 'enabled" ${ }^{10}$ value. However, this approach to 
equilibrium spreads over longer distances as the field is reduced. Within this region the ionisation coefficient falls below its equilibrium value and the multiplication is correspondingly reduced.

Conversely, calculating multiplication from the 'bulk' value of the ionization coefficients, corresponding to the envelope in figure (1), gives an overestimate when the avalanche region is small enough that dead space effects are significant. This is because the local model assumes that ionization can occur in the forbidden dead space and so predicts a larger value than measured. This is most apparent at low bias in the thinnest devices, where the effect of dead space is to increase the voltage for the onset of multiplication over that predicted by the local model.

At high fields a lower limit on this dead space, $d$ can be estimated very crudely as the distance travelled ballistically by a carrier before acquiring from the electric field, $F$, the threshold energy, $E_{T}$ for ionization, determined by considerations of conservation of energy and crystal momentum in the impact ionization process ${ }^{11}$. Hence

$$
d=E_{T} /(e F) \text {. }
$$

In fact carrier energy losses to phonon generation will generally increase this value and, though much less likely, net energy gain by phonon absorption will reduce it, so that the dead space is 'soft'.

When the field is increased to the extent that the dead space becomes an appreciable fraction of the inverse of the enabled ionisation coefficient then, since ionization is prohibited in this dead space region, the measured multiplication is reduced by this effect and the value deduced for the ionization coefficient drops below the envelope. The envelope can thus be interpreted as the ionization coefficient corresponding to a distribution of carriers in equilibrium with the electric field.

This dead space effect is seen in all semiconductor systems investigated to date, including $\mathrm{SiC}^{2}, \mathrm{AlGaAs}^{9}, \mathrm{GaInP}^{12}$, InAlAs ${ }^{13}, \mathrm{InP}^{14}$ and $\mathrm{Si}^{15}$. To account for this effect in any theoretical model of multiplication we must go beyond our local description, in which the ionization coefficient tracks the local field faithfully. We must also acknowledge the region in which the ionization coefficient approaches equilibrium. 
Thus, even in a uniform field an electron's ionization coefficient, $\alpha(x)$ will depend on the distance, $x$ travelled down the field since it was injected. Alternatively, we can describe ionization in terms of a probability distribution function (pdf), $h_{e}(x)$, defined so that $h_{e}(x) d x$ represents the probability that an electron, injected cool at $x$ $=0$, will ionize for the first time in the small interval $(x, x+d x)$. The 'first time' restriction is there because a carrier loses energy on ionising and we need to know when this last happened since it must then traverse a further dead space before ionising again. History matters in nonlocal impact ionisation.

We can expect $\alpha(x)$ and $h_{e}(x)$ to have the following properties. Both will start at zero and rise significantly above this only after the dead space, $d$, since cold carriers cannot ionize. Beyond this region $\alpha(x)$ will approach a constant, 'enabled' value, $\alpha^{*}$, corresponding to an electron distribution in equilibrium with the field, while $h_{e}(x)$ will peak near $x=d$ and at large distances must decay, roughly as $\sim \exp \left(-\alpha^{*} x\right)$, because the decay rate of carriers available for 'first time' ionisation is proportional to their remaining number.

In fact $\alpha(x)$ and $h_{e}(x)$ are related, since the fraction of carriers surviving ionization a distance $x$ down field from injection is given both by $\exp \left(-\int_{0}^{x} d x^{\prime} \alpha\left(x^{\prime}\right)\right)$ and by $1-\int_{0}^{x} d x^{\prime} h_{e}\left(x^{\prime}\right)$. Equating these expressions and differentiating we find that

$$
h_{e}(x)=\alpha(x) \exp \left(-\int_{0}^{x} d x^{\prime} \alpha\left(x^{\prime}\right)\right) .
$$

A little reflection shows that $\alpha(x)$ here must equal the ionisation rate per unit distance of electrons which have escaped ionisation out to a distance $\mathrm{x}$; electrons which have ionised within this interval have an unknown history. 
Relation (6) can be used to deduce a form for $h_{e}(x)$ corresponding to some proposed physical model for $\alpha(x)$. For example, if we suppose that $\alpha(x)=0$ within some dead space, $0<x<d$, jumping to a constant value $\alpha(x)=\alpha^{*}$ for $d \leq x$ then it follows that

$$
\begin{aligned}
& h_{e}(x)=0 \text { for } 0<x<d, \\
& h_{e}(x)=\alpha^{*} \exp \left\{-\alpha^{*}(x-d)\right\} \text { for } d \leq x .
\end{aligned}
$$

Despite its extreme simplicity this 'displaced exponential' model ${ }^{16}$ for the ionization path length pdf has become a favourite for interpreting nonlocal ionization phenomena.

Several authors have addressed the problem of modelling avalanche multiplication including the effects of nonlocal ionization. Okuto and Crowell ${ }^{16}$ were among the first but Hayat et al. ${ }^{17}$ offered perhaps the most transparent exposition, summarised here.

When a carrier precipitates an ionization event both the newly generated carriers and the primary carrier cool in the process and all three must travel their respective dead spaces before ionizing again. Because of this the arguments leading to eqn. (1) cannot easily be adapted. Instead, we imagine a primary electron/hole pair injected cold at $x$ and consider the quantity $Z(x)$, equal to the total number of electrons and holes generated at the series of successive impact ionisation events in a single trial resulting from (and including) the primary electron. We also consider a similar quantity, $Y(x)$ resulting from and including the primary hole. Clearly if we add $Z(x)$ and $Y(x)$ we get twice the total number of resulting electrons hole pairs, so that

$$
M(x)=(Z(x)+Y(x)) / 2 .
$$

In any one trial $Z(x)$ will exceed unity only if the injected electron first ionizes downstream, at $\zeta$, say, where $x<\zeta<w$. This process produces two cold electrons and a cold hole at $\zeta$, each of which go on to generate $Z_{1}(\zeta), Z_{2}(\zeta)$, and $Y(\zeta)$ carriers, respectively. Thus we can write

$$
Z(x ; \zeta)=Z_{1}(\zeta)+Z_{2}(\zeta)+Y(\zeta)
$$

where the LHS represents the quantity $Z(x)$, conditional on the injected electron ionising at $\zeta$,, and the suffixes on the $Z(\zeta)$ on the RHS refer to each cold electron. 
$Z, Y$, and so $M$ will vary from trial to trial and thus are stochastic quantities so that only their distributions and means are significant. In fact the primary electron may ionize for the first time anywhere in $x<\zeta<w$, or may even avoid ionising altogether, so that in evaluating the mean value $\langle Z(x)\rangle$, averaged over many trials, we must allow for this. We find

$<Z(x)>=1-\int_{0}^{w-x} d x^{\prime} h_{e}\left(x^{\prime}\right)+\int_{x}^{w} d \zeta h_{e}(\zeta-x)(2<Z(\zeta)>+<Y(\zeta)>)$.

The second integral on the RHS allows for the primary electron to ionize anywhere in $x<\zeta<w$, with ionization path length pdf $h_{e}(x)$ and the factor of 2 and the loss of the subscripts from eqn. (9) arise because the stochastic variables $Z_{1}(\zeta)$ and $Z_{2}(\zeta)$ have identical distributions. The first integral represents the probability that the primary electron will ionize before it escapes the multiplication region, so that subtracting it from unity represents the converse probability, providing the contribution in the event that the primary electron escapes ionization.

Similar arguments concerning the fortunes of the primary hole lead to the equation

$$
<Y(x)>=1-\int_{0}^{x} d x^{\prime} h_{h}\left(x^{\prime}\right)+\int_{0}^{x} d \xi h_{h}(x-\zeta)(2<Y(\zeta)>+<Z(\zeta)>),
$$

where $h_{h}(x)$ is the ionization path length pdf for holes. Given forms for $h_{e}(x)$ and $h_{h}(x)$ we can solve eqns. (10) using standard numerical techniques, e.g. by iteration or by discretising the integrals and solving the resulting matrix equations, to find the mean value of multiplication from eqn. (8),

$$
<M(x)>=(<Z(x)>+<Y(x)>) / 2 \text {. }
$$

If we use ionization path length pdfs, $h_{e}(x)=\alpha \exp (-\alpha x)$ and $h_{h}(x)=\beta \exp (-\beta x)$ corresponding to uniform, local ionization coefficients (eqn. (7) with $d=0$ ) then it is straightforward to show that eqns. (10) and (11) lead to (3).

While Hayat and Saleh ${ }^{17}$ and many other authors use the displaced exponential approximation, eqn. (7) for the $h_{e, h}(x)$, the nonlocal analysis described above is by no means restricted to this approximation. More realistic Monte Carlo calculations ${ }^{18}$ of ionization path length pdfs for GaAs (fig. 2) show the general features captured in eqn. (7). However, the dead space is strictly a 'sick' space, in which the ionization 
path length pdf rises more gradually to a softened peak, eventually followed by exponential decay. The softening of the peak is associated with the effects of phonon scattering, discussed earlier, and also with the finite rate of ionisation above threshold. The exponential decay, following from the equilibrium achieved by the carrier gas at long distances, is evident. The irregularity in the curves is a consequence of Monte Carlo noise, resulting from limitations on computer power, and is the major drawback of this technique.

\section{Avalanche noise}

The primary photocurrent in an APD is generated by photons which arrive and are absorbed at a Poisson distributed rate, and is carried by electrons and holes with discrete charges, $\mp e$. The resulting fluctuations about the mean current, $I$ have a mean power per unit bandwidth of $P_{N}=2 e I$ corresponding to shot noise ${ }^{19}$. Since this is the spectrum of the squared current fluctuations, avalanche multiplication by a fixed gain, $\langle M>\text { would increase this value to } 2 e I<M\rangle^{2}$.

This result is simplistic for two reasons. The 'white' noise argument is strictly valid only when the current pulses have indefinitely small width. In fact carriers crossing a depletion region generate rectangular current pulses ${ }^{20}$, of width equal to their transit time, $w / v$, for a carrier speed $v$. Consequently the noise is 'pink', with an upper frequency limit of order $w / v \sim 100 \mathrm{Ghz}$ for a $1 \mu \mathrm{m}$ wide depletion region. Unfortunately this high frequency noise suppression does not lead to quieter photodiodes since this transit time effect also makes this an upper bandwidth limit on the gain.

More importantly, the multiplication is not fixed but may take a wide spread of different values for each trial, with a probability distribution not even peaked at the mean value ${ }^{18}$, as shown graphically in fig. 3 . Now in a typical photomultiplication measurement ${ }^{21}$ a stream of carriers is injected optically with a $\mathrm{CW}$ laser beam, chopped at low frequency to help distinguish photocurrent signal from dark, tunnelling current (fig. 4). The photocurrent resulting from each photogenerated carrier is amplified by a stochastic multiplication, $M$. Because of the enormous number of carriers injected in each pulse the variance on the mean gain from pulse to 
pulse is negligibly small, so that the photocurrent in each pulse is effectively multiplied by the mean multiplication, $\langle M\rangle$.

However, in calculating the noise we must average over different trials after squaring the noise current fluctuation amplified by the fluctuating gain, $M$, introducing a factor $<M^{2}>$. The noise power per unit bandwidth in an APD thus becomes $P_{N}=2 e I<M>^{2} F$, where

$$
F=<M^{2}>/<M>^{2}
$$

is the excess avalanche noise enhancement factor ${ }^{6,7}$.

Noise can also be measured using phase sensitive detection ${ }^{21}$ to eliminate dark current contributions (fig. 4). Chopped light is detected in the APD test structure and the resulting signal is band pass filtered. This eliminates the DC mean multiplied photocurrent and $1 / f$ noise and also makes the frequency range accessible to the following square law power meter before detection at the chopping frequency. By comparing the noise power measured at a known value of mean multiplication and current with that measured on a $\mathrm{Si}$ photodiode operated at unit gain, in which unmultiplied shot noise dominates, the effective bandwidth of the system can be factored out to extract the excess avalanche noise factor.

McIntyre $^{8}$ was the first to calculate this factor, $F$, within the local approximation, whose limitations he was at pains to point out. Using a technique similar to that which led to eqn. (1) and ascribing full shot noise to the current generated by each ionization event he showed that, for pure electron injection, the noise enhancement factor is given by

$$
F_{e}=k<M_{e}>+\left(2-1 /<M_{e}>\right)(1-k)
$$

and for pure hole injection

$$
F_{h}=<M_{h}>/ k+\left(2-1 /<M_{h}>\right)(1-1 / k),
$$

where $k=\beta / \alpha$. Two features are particularly worth noting in these expressions.

Firstly, for a given value of multiplication the excess avalanche noise can be reduced by choosing a material with a small ratio of secondary to primary carrier ionization 
coefficients. This principle has guided the design of low noise APDs for around 40 years. However, its naive acceptance and cavalier application outside the restricted domain clearly identified by McIntyre may have impeded the development of high performance APDs, for reasons which will become clear.

At low electric fields electrons and holes can have widely differing ionization coefficients in the same material, because of the differing details of the band structure and of phonon scattering processes which dominate their low field transport. However, as the field increases so the increasing ionization coefficients approach one another, for the following reason. At the highest fields carriers have little time to scatter off phonons before acquiring their ionization threshold energies and reach threshold in little more than the ballistic dead space given by eqn. (5), ionising shortly afterwards. In this limit the ionization coefficients are given approximately by the inverse of these distances for electrons and holes. Since ionization threshold energies for electrons and holes normally lie between one and two times the band gap ${ }^{11}$, and so are rarely too different, neither are the ionization coefficients at these high fields.

It follows that a value of $k$ much less than unity, required for low excess noise following pure electron injection, or much greater than unity, required if pure hole injection is adopted, cannot be achieved at high fields. But at low fields ionization is drastically reduced and respectable values of multiplication can be achieved only in wide devices, where the multiplication has enough space to grow. Unfortunately, long multiplication regions increase the time taken for the avalanche process to build up and decay because of the long carrier transit times. Moreover, impact ionization is more sensitive to temperature at low fields, since carriers scatter many times before ionising, losing or gaining the energy of an optical phonon at a rate which depends on temperature via the phonon population. Furthermore, a long multiplication region needs an inconveniently large external bias to maintain electric field.

These evident conflicts between the requirements for low noise, predicted on the local model $^{8}$, and other desirable performance characteristics can only have helped relegate the APD to second class status in comparison with, say pin-FET modules, where the functions of detection and pre-amplification are distributed between the two components and the design conflicts described above do not arise. 
Returning to eqns. (13), their second notable feature is that the excess avalanche noise is predicted to depend only on the multiplication and ionization coefficient ratio, and not on multiplication region width. While this prediction may apply to wide multiplication regions fig. 5 shows that it fails when these regions approach the submicron scale ${ }^{22}$. In fact at a fixed value of mean multiplication, the excess noise factor in GaAs pin diodes falls significantly as the device width is reduced and the electric field is increased to maintain multiplication ${ }^{23}$. The faint lines in fig. 5 are drawn by plotting the results of eqns. (13) against those of (3) and so constitute the predictions of the local model. While this noise reduction effect might have been expected for pure hole injection, since in GaAs $\alpha \beta$ decreases towards unity with increasing field, the corresponding avalanche noise for pure electron injection should then increase, by the same token. The observed reduction for both hole and electron injection was therefore quite unforseen.

The reason for this behaviour, which is contrary to the predictions of eqn. (13), is as follows $^{22,23,24}$. In wide multiplication regions, operating at low fields, the ionization coefficients are 'exponentially' small (the linear decrease of $\ln (\alpha)$ with inverse field in fig. 1 illustrates this loose terminology), so that the mean electron and hole ionization path lengths, equal to $1 / \alpha$ and $1 / \beta$, are large. As the multiplication region is narrowed then, in order to maintain multiplication, the field must increase to maintain $\alpha w$ and $\beta w$, the probabilities of ionising at least once in the multiplication region. In this process the dead spaces $d_{e}$ and $d_{h}$ also decrease, roughly according to eqn. (5), but they do so algebraically and much more slowly than the exponential decrease of $1 / \alpha$ and $1 / \beta$. As a consequence ionization becomes increasingly confined to a small region centred on a distance roughly an ionization dead space downstream from carrier injection. This localisation with increasing field is evident in the simulations of fig. 2. But the noise factor is a measure of the randomness in multiplication, which in turn is caused by the spatial randomness in the ionization process. By localising ionization events and making the multiplication process more deterministic we reduce this randomness and hence reduce the avalanche noise. 
The fall in excess avalanche noise as the multiplication region shrinks has now been observed in a wide range of materials, including $\mathrm{GaAs}^{25}, \mathrm{AlGaAs}^{26,27,28}, \mathrm{InGaP}^{29}$, $\mathrm{InP}^{30}$, InAlAs ${ }^{30,31}$ and $\mathrm{Si}^{32}$. Fig. (6) shows measurements of excess avalanche noise in Si pin diodes resulting from both pure electron injection and also from 'mixed' injection. In the latter case some light is absorbed within the multiplication region itself, so that both electrons and holes go on to multiply, although in this case the holes initiate most of the multiplication. The noise is evidently lower for pure electron injection, because at low fields $\alpha>\beta$ in $\mathrm{Si}$, and for the mixed (impure hole) injection the noise falls as the multiplication region narrows. However, in the case of pure electron injection the noise is largely independent of multiplication region width. This results from competition between the increasing importance of dead space, as the multiplication width falls, and the increase in $\beta / \alpha$ as the electric field is increased to maintain multiplication in the thinner multiplication regions.

The advantages of reduced noise combined with increased speed and lower operating voltage resulting from narrow multiplication regions are now clear for modern III-V APDs. Indeed, it would seem that pragmatic reductions were made in the sizes of III$\mathrm{V}$ devices by commercial manufacturers (as evidenced by the reduced breakdown voltages on their published data sheets) even before it became publicly apparent why shrinking the multiplication region would not necessarily increase noise.

The problem of how to calculate noise enhancement factor when ionization is nonlocal was addressed by Van Vliet et al. $^{33}$. However Hayat and Saleh again give the clearest exposition ${ }^{17}$, an extension of their technique for calculating the mean multiplication. By squaring the quantities $Z(x, \zeta)$ (eqn. (9)) and the similarly defined quantity for hole injection, $Y(x, \zeta)$ they were able to construct integral equations for $\left\langle Z^{2}(x)\right\rangle$ and $\left\langle Y^{2}(x)\right\rangle$, from whose solutions the excess noise factor

$$
F(x) \equiv \frac{\left\langle M^{2}(x)\right\rangle}{\langle M(x)\rangle^{2}}=\frac{\left\langle Z^{2}(x)\right\rangle+\left\langle Y^{2}(x)>+2<Z(x)\right\rangle\langle Y(x)\rangle}{(<Z(x)>+<Y(x)>)^{2}}
$$

can be deduced. Application of this nonlocal model for noise in Si accounts well (fig. (6)) for the combined effects of increasingly important dead space and converging $\alpha / \beta$ ratio in the increasing electric field. 
Unfortunately, a high field can lead to a high tunnelling current, which also gets multiplied and increases parasitic noise, unless we work with wide band gap materials. To this end multiplication and noise were studied ${ }^{34,35}$ in a range of $\mathrm{Al}_{\mathrm{x}} \mathrm{Ga}_{1-}$ ${ }_{x}$ As alloys, conveniently close to lattice match with GaAs. As well as the expected reduction in noise with reducing multiplication region width an interesting sharp increase in the $\alpha \beta$ ratio just below $x=80 \%$ corresponding to an abrupt fall in $\beta$ becomes apparent in this material. We may speculate that this is perhaps associated with the closure of an impact ionization channel as the band structure changes and a threshold disappears ${ }^{11}$. Fig. 7 shows the very low noise ${ }^{36}$ achievable in this material as the multiplication length is reduced. These findings are supported by the work ${ }^{27}$ of Zheng et al., who have also observed low noise at an Al concentration of $90 \%$. The combination of wide band gap to suppress tunnelling, close match to a GaAs substrate, large $\alpha \beta$ ratio and further noise reduction from the effects of dead space makes this a particularly interesting material for APDs. Thus, if ever lattice matched dilute nitride $\operatorname{GaInAs}(\mathrm{N})$ can be demonstrated to absorb at telecommunications wavelengths the $\mathrm{GaAs}$ based $\mathrm{GaInAs}(\mathrm{N}) / \mathrm{Al}_{0.8} \mathrm{Ga}_{0.2} \mathrm{As}$ APD could become an attractive proposition. Alternatively, GaInAs quantum dots might also provide the absorption medium, given sufficient optical absorption, perhaps with the help of an optical cavity.

The effects of noise reduction associated with dead space are also seen in the even wider band gap material $4 \mathrm{H} \mathrm{SiC}^{37,38}$, raising the possibility of low noise, visible blind, UV APDs. Fig. 8a shows the noise measured on 4H SiC pin APDs resulting from $230 \mathrm{~nm}$ optical excitation. This provides electron injection contaminated with some mixed injection in the high field region, resulting from incomplete optical absorption in the p-type cap. Fig. $8 \mathrm{~b}$ shows the results of $365 \mathrm{~nm}$ excitation, providing slightly impure hole injection. Reducing the multiplication width from $0.285 \mu \mathrm{m}$ to $0.105 \mu \mathrm{m}$ improves the "effective McIntyre ratio", $(\beta / \alpha)_{\text {eff }}$ (equal to the ratio $\beta / \alpha$ of the curve deduced from eqns (3) and (13) which best fits the measurement) for avalanche noise following electron injection from $k=2.7$ to 0.8 . By contrast, the effective ratio, $(\alpha \beta)_{\text {eff }}$ for hole injection remains approximately unchanged at around $k=0.1$. The 
fact that the products, $(\beta / \alpha)_{\text {eff }} \times(\alpha / \beta)_{\text {eff }}$, are considerably less than unity for both device lengths is evidence that dead space effects are significantly reducing noise.

Perhaps the most spectacularly nonlocal ionization behaviour was $\operatorname{seen}^{39,40}$ in $\mathrm{Hg}_{0.7} \mathrm{Cd}_{0.3} \mathrm{Te}(\mathrm{CMT})$. In this material, with a direct band gap at $\Gamma$ of $E_{g}=0.29 \mathrm{eV}$, the heavy hole/conduction band effective mass ratio is around 30. Because of their high mass, heavy holes are available close to the top of the band with momentum large enough to participate in impact ionisation of electrons with kinetic energy little more than the band gap. Consequently the ionization threshold energy for electrons lies at $\sim 0.29 \mathrm{eV}$ above the conduction band minimum, well below the $L$-minimum, which lies some $1.2 \mathrm{eV}$ higher. Moreover, electrons can accelerate rapidly to threshold, while the high rate of hole scattering, resulting from the high density of final scattering states in the heavy hole band and degenerate light hole band, suppresses hole heating and ionization. Furthermore, not only is the $\beta / \alpha$ ratio extremely small as a consequence, but the momentum randomising process of inter-subband scattering is absent for electrons at energies below ionization threshold and their scattering is then largely due to the, predominantly forward directed, polar optical phonon scattering within the $\Gamma$-valley.

As a result, electrons accelerate quasi-ballistically to ionization threshold each time they traverse a ballistic dead space and their numbers double at each of these highly deterministic ionization events, much in the manner of electrons in a photomultiplier tube. Consequently the mean multiplication increases almost exponentially with applied bias and the excess noise factor stays close to unity for gain values of up to $\langle M\rangle \sim 1000$. The near unity noise figure is very impressive and is strong evidence of nonlocal behaviour, since the local model predicts a lower limit (eqn. (15)) of $F=2$. It seems likely that the feedback process due to hole ionization is sufficiently inhibited that the exponential increase of gain with bias is maintained up to these high values of multiplication and that true breakdown (resulting from the divergence discussed earlier which requires feedback from hole ionisation) is indeed suppressed, at least up to these values of field. 
Similar behaviour is predicted ${ }^{41}$ in InSb, and for the same reasons. A narrow band gap leads to a large heavy hole/electron mass ratio, together with an increased separation between the conduction band edge and the next highest minimum. These conditions are also met in InAs, where electron initiated avalanche gain greatly exceeds that initiated by holes ${ }^{42}$, suggesting that $\alpha>\beta$, and the excess noise factor $^{43}$ for electron initiated multiplication remains below 2, providing the strongest evidence for multiplication dominated by dead space.

The effects of nonlocal ionization on multiplication and noise are observed widely, there seems to be little doubt that they are real and can be exploited. Further, unforseen benefits for APDs from nonlocal ionization will be discussed in later sections.

\section{Simulation techniques}

The ionization path length pdfs $h_{e}(x)$ and $h_{h}(x)$, often summarised in the displaced exponential model of eqn. (7) by a dead space and an enabled ionisation coefficient, occupy the key positions in nonlocal models of multiplication taken by the ionization coefficients in the local picture. In this section we examine how their structure may be determined and understood and how they may be used. We also review some alternative techniques for modelling the avalanche multiplication process.

In real APDs, and even in experimental test structures, electric fields are often nonuniform, perhaps because of the diffusion of dopant or carriers from the $\mathrm{p}^{+}$and $\mathrm{n}^{+}$ caps or because of residual doping in the depleted multiplication region. McIntyre ${ }^{10}$ proposed a way of extending the displaced exponential model for ionization path length pdfs restricted to uniform, 1-D electric fields to account for such 1-D nonuniformities. He also showed how to generalise the work of Hayat ${ }^{17,44,45}$ et al. to calculate multiplication and noise in such one dimensionally nonuniform structures.

At the other extreme the Monte Carlo technique ${ }^{46}$ provides an essentially exact solution of the Boltzmann transport equation for semiclassical charge carriers. It follows the trajectories of individual particles as they move around in real- and momentum-space under the influence of the driving electric fields and accounts for 
the random influence of scattering with the help of a random number generator. This enormously powerful technique is limited only by the capacity of the computer used to perform the simulation. At its best, using a band structure calculated with an empirical pseudopotential adjusted to fit measurement, a full range of phonon scattering processes, first principles calculations of impact ionization rates, etc., it represents the gold standard for modelling (at least when scattering processes can be described by Fermi's golden rule and other quantum phenomena are unimportant). It is then the numerical equivalent of an experiment and its results can be almost as difficult to interpret. It includes automatically the physics behind nonlocal impact ionization, it was used to generate the pdfs shown in fig. 2 and can be used to simulate the complete avalanche process, including its evolution in time and allowing for the awkward 2-D or 3-D structures used in real devices. If required it can be linked to a thermal transport simulator to account for effects of heating on carrier transport via increased phonon generation and can include the effects of avalanche carrier space charge on the electric field profile. However, it can also soak up as much computing power as is available and its use can require considerable, dedicated expertise. Techniques less sophisticated and demanding are therefore also welcome, provided their simplicity does not 'throw out the baby with the bath water'.

Ridley's 'Lucky Drift' model ${ }^{47,48,49}$ was remarkably successful in predicting the material and field dependences of local ionization coefficients, given its extreme simplicity. Ridley ${ }^{47}$ observed that, while phonon scattering is the common source of both momentum and energy relaxation in ensembles of charge carriers, an artificial separation of these two relaxation processes was convenient. In his picture carriers acquire energy by 'drifting' down the field, a process determined by momentum relaxation, while their energy loss to phonons is described by less frequent, random 'supercollisions' in which carriers lose all of their energy and are condemned to begin their journey to ionization threshold all over again.

The success of this strategy in modelling local ionisation coefficients did not extend to the nonlocal regime ${ }^{50}$. Fig. 9 shows calculations ${ }^{51}$ of $h(x)$ using the principles of Lucky Drift for a simple model material, with a parabolic band, a hard ionization threshold and only optical phonon scattering, which also randomises momentum. The model gives rise to pathological behaviour in $h(x)$, with a $\delta$-function at the dead space, 
followed by a plateau and more, though less obvious, unphysical behaviour further downstream. While the simple assumptions of Lucky Drift describe well the mean value of the ionization path length and its inverse, the ionization coefficient, they appear to be far too simplistic to describe the details of its pdf.

What is wrong here is the assumption that a carrier loses all of its energy in one catastrophic scattering event, rather than distributing it to the phonon gas one quantum of phonon energy, $\hbar \omega$, at a time, and even gaining this energy on occasion. The Fokker-Planck technique ${ }^{52}$ provides a method for calculating $h(x)$ which retains this important physics, while discarding much of the unnecessary complexity. This technique is based on two assumptions, namely that momentum relaxation due to phonon scattering is so fast that the carriers' phase space distribution function can be considered to depend only upon energy, rather than momentum, and that the energy gained or lost on phonon scattering is small on the scale of things. The former assumption is not too bad, especially at the high fields encountered in impact ionization. In this case energetic carriers have a wide choice of scattering destinations among the (sub-) bands in most group III-V and IV semiconductors and the forward momentum directionality of polar optical phonon scattering is lost (although this assumption breaks down when polar optical phonon scattering dominates within the $\Gamma$-minimum, such as in CMT, InSb and InAs). The latter assumption is quite good because invariably $\hbar \omega<<E_{T}$.

The Fokker-Planck equation can be derived ${ }^{53}$ from the Boltzmann transport equation by expanding the distribution function in momentum as far as the first two Legendre polynomials. The second is then eliminated by replacing the square of the momentum by its mean, in order to truncate the series, and the phonon energy is treated as an infinitesimal, with suitably adjusted scattering rates. Because the carrier transport in position- and energy-space is (quasi-) continuous a diffusion-drift type of differential equation in both position and energy results, which can be solved by standard numerical techniques. Fig 9 also shows a Fokker-Planck calculation of $h(x)$ using the same parameters as used for the Lucky Drift calculation. The Fokker-Planck technique would seem to have potential for direct evaluation of the avalanche process in realistic APD structures, in which the radial, as well as the longitudinal motion of 
carriers and dependence of electric field is important. The results are far more realistic than those of Lucky Drift and are very close to those of our equivalent 'simple' Monte Carlo ${ }^{54}$ calculation.

This latter is a Monte Carlo technique stripped down to its bare essentials; single parabolic band (regardless of the complexity of the band structure at high energies), scattering by dispersion free, momentum randomising optical phonons, with ionization threshold energy, softness of threshold, carrier mass and electron-phonon coupling constant chosen to fit experiment. However, the method does include the acquisition of energy and momentum from the electric field, punctuated and moderated by random phonon scattering which introduces momentum relaxation, quantised energy gain and loss and hence the diffusion process, in a realistic way which eludes analytical treatment. Despite its extreme simplicity and flagrant distortion of the known facts about semiconductor band structures this simple technique has proved remarkably powerful as a tool for interpreting and understanding measurements on both multiplication and noise in the nonlocal regime and also, as we shall see later, on device speed.

An even simpler numerical technique is the 'Random Path Length' (RPL) method ${ }^{55}$, a kind of poor man's Monte Carlo model. It takes the pdfs of ionization path length as its starting point, maybe using displaced exponentials or perhaps something a little more fancy, generated by a more sophisticated Monte Carlo calculation, and uses a random number generator to construct a sequence of ionization events to simulate a multiplication 'trial'. By combining the results of many such trials one can calculate suitable running averages to study a variety of statistics. As the number of trials increases this method gives identical results to the integral equation methods ${ }^{10,17}$, devised for calculating multiplication and noise, since it makes the same assumptions. However, it has the added advantages of conceptual simplicity and the capacity to evaluate a variety of other statistics as running averages in the same numerical experiment. Because of its modest demands we have found this technique extremely useful and flexible for interpreting measurements and understanding avalanche multiplication in the nonlocal regime.

\section{Speed}


RC time constants and parasitic effects apart, the intrinsic speed of an APD is limited by the time taken by the avalanche current to build up and decay, controlled by the time for ionising carriers and their offspring to travel back and forth across the multiplication region before finally exiting. We can therefore expect the avalanche response time to increase with multiplication region width and with the mean value of multiplication and to vary inversely with the carrier speeds. However, if multiplication results only from ionization by carriers of the type which are injected as primaries, then these carriers will traverse the multiplication region as a group within one transit time. The avalanche will then be over by the time it takes any carriers of the opposite type, generated by impact ionization, to make the return journey. We can therefore also expect APD speed to depend sensitively on $k$. These trends were confirmed in the calculations of Emmons ${ }^{56}$ and of Kuvås and Lee ${ }^{57}$. Both of these early treatments assumed uniform electric fields, local ionization and constant drift velocities for the charge carriers, ignoring the effects of diffusion.

Hayat $^{58,59}$ et al. adapted their integral equation technique ${ }^{17}$ to predict the current impulse response of an APD, the mean value of evolving avalanche current, and also of its second moment, following primary carrier injection, taking account of the effects of dead space. They did this by calculating the time dependence of the concentration of carriers in the high field region, assuming that these carriers travel with constant velocities, and they used the simple displaced exponential model of eqn. (7) to describe their ionization path length pdfs. They concluded that the effect of dead space is to slow down the avalanche process. Dead space denies carriers access to some of the high field region for the purposes of impact ionization. To maintain the same degree of multiplication carriers must travel beyond the dead space to ionize, postponing ionization and prolonging the avalanche.

$\mathrm{Ng}^{60}$ et al. studied the mechanisms responsible for this reduction of APD speed from the effects of dead space using the RPL technique. Again assuming constant carrier velocities they concluded that the avalanche response time is determined by competition between two processes. The narrowing of the ionization path length pdf and the associated increased determinism in the multiplication process actually narrows the multiplication distribution, exemplified in fig. 3, by reducing the probability of generating higher values of multiplication. This both reduces noise and 
also speeds up the avalanche process, on average, since the shorter multiplication chains take correspondingly shorter to complete. However, the postponement of individual ionization events noted by Hayat et al. ${ }^{17}$ has the result that any given value of multiplication $M$, which may be produced in a variety of ways by successive ionization of either electrons or holes, or their combination, must rely more on processes in which electrons and holes travel sequentially back and forth across the device, delaying the exit of the final carrier in the chain. It turns out that this latter effect wins and dead space effects are predicted to slow down the avalanche process, at least according to the assumptions of this model.

So far we have discussed models which have assumed, quite reasonably, that, at the high electric fields needed for avalanche multiplication, carriers travel always at their saturated drift velocities and that the effects of carrier diffusion can be ignored. However, Monte Carlo simulations of the spatial and temporal statistics of the ionization process by Plimmer ${ }^{61}$ et al. suggest that this may not be such a good approximation. Not only does diffusion appear to be significant but those carriers which ionize at short distances down the field, rather than further downstream, appear to arrive at these 'spatially early' ionization events with unusually high average velocities.

Plimmer $^{61}$ et al. used a moderately sophisticated model for the band structure in their Monte Carlo simulation, involving three analytically described conduction subbands, at $\Gamma, X$ and $L$. Hence, it is tempting to ascribe this velocity enhancement to the conventional velocity overshoot mechanism which leads to intervalley transfer and Gunn oscillation. However, the effect is also seen in the simple Monte Carlo model ${ }^{54}$, which uses only a single parabolic conduction band valley and has no intervalley transfer, so this cannot provide the explanation. Moreover, it is also predicted by the Fokker-Planck technique ${ }^{53}$, in which momentum scattering is supposed fast enough that the carrier distribution in the bands depends only on energy. This would therefore also appear to rule out ballistic transport as a general explanation, although this process is likely to operate at the earliest times during a carrier's trajectory. The effect is also predicted by the most sophisticated Monte Carlo simulation available to us $^{62}$, and we believe that it is real. 
Fig. 10 shows the effect most clearly in terms of the quantity $h(x, t)$, the pdf of ionization events in both space and time, calculated ${ }^{53}$ using the Fokker-Planck technique for a model semiconductor. This $h(x, t)$ represents the probability, per unit time and per unit distance, that a carrier, injected cold at position $x=0$ at time $t=0$, will ionize for the first time at $(x, t)$. The structure shows a ridge in the $x$ - $t$ plane whose amplitude decays roughly exponentially with increasing $x$ and $t$ and which also decays away rapidly on either side of the ridge. The line of this ridge intersects the position axis at a distance which represents the dead space and the decay on either side of the ridge corresponds to carrier diffusion. However, perhaps the most interesting aspect is the fact that the 'dead time' to ionization is negligibly small on this diagram. This suggests that carriers arrive at the dead space extremely fast and then travel downstream to their ionization events at a constant velocity, given by the gradient of the ridge projected onto the $x$ - $t$ plane. Fig. 11 shows a contour plot of the same quantity, calculated ${ }^{63}$ using the Simple Monte Carlo technique and showing similar behaviour.

If this effect, of velocity enhancement to early ionization, is real then its consequences on APD speed are quite promising. By modelling the distribution of exit times of the last avalanching carrier from a multiplication region ${ }^{64,65}$, also known as the 'avalanche duration', and also by modelling the mean current impulse response ${ }^{66}$ Hambleton et al. have shown that the effect is to speed up the avalanche process significantly in short devices. Monte Carlo simulations suggest that this speed enhancement more than compensates for the reduction in APD speed due to the effects of dead space alone ${ }^{54,55}$.

The integral equation technique has been extended ${ }^{63}$ to calculate the current impulse response, and also its standard deviation, using arbitrary two dimensional pdfs, $h(x, t)$ such as illustrated in figs. 10 and 11. Results, shown in fig. 12 confirm that diffusion, though apparent in $h(x, t)$, has little effect on the mean current impulse response, since the values of diffusion coefficient needed to make an appreciable difference are unrealistically large. The results also support the improvements in APD speed predicted by Monte Carlo $54,61,62,63,65,66$. 
Hayat and Dong ${ }^{67}$ derived an integral equation approach to calculate avalanche duration, which they termed the 'random response time', also studied numerically by Hambleton $^{64,65}$ et al. This avalanche duration is a particularly interesting statistic in digital optical fibre communications since we are there concerned with how photocurrent pulses spread and merge. Avalanche duration is a stochastic variable and Hayat and Dong showed how to calculate its pdf for use in estimating its mean value and the mean of its inverse, related to the APD bandwidth. Their technique was restricted to constant carrier velocities and, although it allowed for an arbitrary ionization path length pdf, these authors used the technique only to assess the pdf of avalanche duration for the case of local ionization. This approach was subsequently generalized by $\operatorname{Sun}^{68}$ et al. to evaluate the joint pdf of the avalanche duration and the avalanche multiplication, allowing determination of the statistical correlation between these two quantities.

A further generalisation ${ }^{69}$ of the technique to handle arbitrary two dimensional pdfs, $h(x, t)$ shows that the resulting pdfs of avalanche duration are also relatively insensitive to the effects of diffusion, as seen in calculations on current impulse response ${ }^{63}$. The technique also shows the same speed improvement in the avalanche duration, resulting from enhanced speed to early ionisation, as in other statistics and using other methods.

This enhancement in the speed of a carrier to early ionization, although predicted by a number of different techniques, eluded physical explanation for some time. Its origin now seems obvious with hindsight. Carriers which ionize early in their trajectories have had a privileged history; they must have scattered infrequently on their journey downstream to ionization. Since the rates of phonon absorption and emission are proportional to $n$ and $n+1$, where

$$
n=(\exp (\hbar \omega / k T)-1)^{-1}
$$

is the phonon occupation number, the carrier loses an energy, on average, of $\hbar \omega /(2 n+1)$ each time it scatters off a phonon. If we suppose that $N_{l}$ represents the average number of scattering events by phonons encountered by carriers which go on to ionize a distance $l$ down stream from their injection point, then, assuming for 
simplicity that carriers ionize immediately on reaching threshold, simple energy balance arguments show that $E_{T}=e F l-N_{l} \hbar \omega /(2 n+1)$, so that

$$
N_{l}=\frac{e F(2 n+1)}{\hbar \omega}(l-d) .
$$

Thus, carriers whose ionization path length, $l$ is only slightly larger than the ballistic dead space, $d$, given by eqn. (7), will, on average, have scattered very infrequently since they were injected. This infrequent scattering is reflected in increased drift velocity prior to ionising of this privileged class of carriers, leading to the effects observed in our Monte Carlo 'experiments'. We should point out that while we have seen these enhanced velocities in all of our simulations, they have not yet been observed unequivocally in real experiments.

The dynamics of the growth and decay of populations of carriers born of earlier generations have analogies in those of living populations and it is not surprising that some of the consequences are similar. By examining the behaviour of the integral equations describing the evolution of carrier populations in a uniform multiplication region, Hayat and Saleh ${ }^{17}$ showed that, below breakdown, both the mean value of the avalanche current and its mean square deviation from this mean current decay asymptotically as $\sim \exp (-\varkappa)$, so that the standard deviation in current decays as $\sim \exp (-\varkappa / 2)$. Here the Malthusian parameter ${ }^{70}, \gamma$ depends only on the ionization path length pdfs in position and on the multiplication region width, and not on the initial conditions.

Indeed, we observe this exponential decay of current at long times in our numerical (Monte Carlo and RPL) simulations. Groves et al. ${ }^{71}$ showed analytically that this effect is rather more general, occurring in any 1-D nonuniform field profile and for general models of ionization event pdfs. In particular the velocity enhancement effect speeds up the process when dead space represents a significant fraction of the avalanche width. These authors showed that this Malthusian decay also dominates the long term behaviour of the avalanche duration, explaining our observation of this in our numerical simulations of this process ${ }^{69}$. 


\section{Temperature stability}

III-V semiconductor band gaps shrink with temperature. For example, the GaAs band gap falls by around 5\% on warming from liquid He to ambient. Ionization threshold energies are likely to change in a similar manner, since they are determined by considerations of conservation of energy and crystal momentum within the band structure framework ${ }^{11}$. While a reduction in ionisation threshold energy would serve to increase the ionisation coefficient, the accompanying changes in band structure slope and curvature will also help determine the shift in ionisation threshold energy ${ }^{11}$ and the net trend is not clear.

Electron-phonon scattering thermalises and cools the populations of carriers heated in strong electric fields. The rates of optical phonon absorption and emission by charge carriers are given respectively by $n C$ and $(n+1) C$, where the factor $C$ depends on the band structure and the electron phonon coupling strength and $n$ is the optical phonon occupation number, given by eqn. (15). But phonons become more populous with temperature. In GaAs, where the optical phonon energy is around $30 \mathrm{meV}, n$ increases from effectively zero at liquid He to $n \sim 0.43$ at ambient temperature, so that the emission rate increases by $43 \%$ over this range and the absorption rate also increases, from effectively zero at liquid $\mathrm{He}$ to $30 \%$ of the emission rate at room temperature. Because of this increase in scattering rates with temperature the associated cooling of carriers in high electric fields grows stronger as the lattice is heated. So, counter intuitively, heating the lattice cools the charge carriers in a high field, because at high fields the charge carriers are much hotter than the lattice, whose heating serves to enhance its interaction with the carriers. The net effect is to inhibit the transport of carriers to ionisation threshold and reduce ionisation coefficients. Indeed, Ma et al. ${ }^{72}$ observe the saturation expected in the ionization coefficients at low temperatures as the optical phonon occupation number approaches zero.

At large values the mean multiplication is particularly sensitive to the ionization coefficients. For example, when $\alpha=\beta$ in the local model it is given by $<M>=(1-\alpha w)^{-1}$, so that $\frac{d<M>}{d \alpha}=<M>^{2} w$ and the logarithmic temperature sensitivity is given by $\frac{1}{<M>} \frac{d<M>}{d T}=<M>\alpha w \frac{1}{\alpha} \frac{d \alpha}{d T}$. At appreciable values of 
multiplication, when $\alpha w$ is close to unity, the temperature sensitivity of the mean multiplication is thus proportional to that of the ionization coefficient, but magnified by the mean gain.

Measurements on the $\mathrm{Al}_{0.6} \mathrm{Ga}_{0.4} \mathrm{As}$ material system show that the temperature sensitivity is much reduced for devices with short multiplication regions ${ }^{73}$. Fig. 13 shows measurements ${ }^{74}$ of mean multiplication, plotted on a log scale to expand the range, against bias, for a range of temperatures and of device lengths. The shorter devices show considerably reduced variation of multiplication with temperature. We believe this results from that fact that as the device length is reduced the ionization path lengths must also be reduced (by increasing the field) to maintain the same degree of mean multiplication. The reduced temperature dependence follows simply because carriers then have a shorter distance in which to scatter off phonons before ionising. Similar effects are seen in short $\mathrm{GaAs}_{\text {structures }}{ }^{75}$ and also in $\mathrm{Si}^{76}$, though the effect of temperature is weaker in this material, possibly ${ }^{77}$ because of its softer ionisation threshold.

\section{Above breakdown}

When an APD is biased above breakdown at least one of the many electron-hole pairs injected in an optical pulse may give rise to a chain of multiplication events which, in principle could go on indefinitely. Such 'infinite' chains dominate the mean multiplication in any large ensemble of trials and, in the absence of other limiting factors, the mean multiplication is then predicted to diverge (as in eqns. (3)). The resulting current then continues to increase until other effects intervene to quench the flow. However, this does not mean that all trials performed under conditions 'above breakdown' will lead to a divergent multiplication; many such multiplication chains will eventually fizzle out, giving a finite value of multiplication for that trial. The breakdown probability, $P_{b}$ for a single trial then becomes the interesting quantity above breakdown in an APD.

The photocurrent generated by a single photogenerated electron-hole pair amounts to $^{20} q v / w \sim 10^{-8} \mathrm{~A}$ and lasts for only $w / v \sim 10^{-11} \mathrm{~s}$ in a $w=1 \mu \mathrm{m}$ wide multiplication region. If the photocurrent is to be detected by conventional electronic circuitry then 
the primary electron-hole pair must go on to enjoy a multiplication of a few orders of magnitude, corresponding to a virtually endless multiplication chain. The breakdown probability therefore effectively determines the probability of detecting a single absorbed photon in an APD operated in Geiger mode, above breakdown. Such Single Photon Avalanche Diodes (SPADs) have application in quantum key distribution in secure systems, since observation by an eavesdropper of the quantum state of the photon can affect this state in a way detectable by the legitimate observer. These devices also have a number of other uses; for example in precision range finding, satellite laser ranging, deep space laser communication, time resolved photon counting spectroscopy and quantum cryptography.

Breakdown at low electric fields is an advantage in SPADs, since high electric fields can strip charge carriers from deep traps, perhaps captured there from a previous avalanche. Such carriers may then go on to initiate another avalanche, which, if detected, would constitute a 'dark count', registered erroneously as a detected photon. However, SPADs with reduced multiplication region widths will require increased electric fields to maintain breakdown. Moreover, since ionisation is denied in the dead space, avalanche breakdown will be postponed, requiring a further increase in electric field. Thus, while short SPADs have obvious speed advantages, because of their reduced transit time, the associated enhancement in dead space effects is not of obvious advantage to SPAD operation.

Once breakdown is reached the next important consideration is the rate at which $P_{b}$ increases with field; a rapid increase is an advantage if dark counts are to be avoided. In the local model breakdown probability was studied by Oldham ${ }^{78}$ and McIntyre ${ }^{79}$. They showed that $P_{b}$ is zero precisely at breakdown and as the ionization coefficients increase with the field above breakdown so the breakdown probability increases first linearly with ionisation coefficients and then approaches unity asymptotically. Thus, in most trials at fields high above breakdown threshold the current increases until limited by external factors. The initial, linear rate of increase was predicted to be faster for a larger ratio of primary to secondary ionization coefficient and these local predictions were supported by measurements on $\mathrm{Si}^{78}$. 
McIntyre later extended his local theory ${ }^{79}$ to include nonlocal behaviour ${ }^{10}$. Using this nonlocal technique Wang et al. ${ }^{80}$ predicted that in SPADs with avalanche regions composed of GaAs, InAlAs or InP the breakdown probability increases more slowly just above breakdown threshold in narrower avalanche regions. These authors ascribed this result to the effects of dead space. However, in a more detailed study $\mathrm{Ng}$ et al. ${ }^{81}$ showed that allowing for dead space actually increases this initial linear rate of growth of $P_{b}$, but that this effect competes with that of the convergence of the ionization coefficients at high fields. This convergence tends to reduce the rate of growth of $P_{b}$ in a SPAD designed so that the primary ionisation coefficient is larger ${ }^{78}$, and this effect wins the competition in the specific cases studied by Wang et al. ${ }^{80}$.

Also of interest is the rate at which the avalanche current increases with time when the current in a particular trial happens to grow very large. The sooner the event can be detected and registered as a 'count' the sooner the system can be reset to detect the next incoming photon and the greater is the system band width. To register as a count the current must exceed an arbitrary, predetermined threshold, say $1 \mathrm{~mA}$ (corresponding to a gain of $M \sim 10^{5}$ ). This threshold is also sometimes confusingly referred to as the 'breakdown threshold'. In order to avoid confusion with the 'breakdown threshold' associated with the divergence of eqns. (3) we shall instead refer to this as the 'decision threshold'.

Of course, the avalanche current above breakdown is also a stochastic variable and is just as susceptible to random fluctuations as it is below. However, in this case we are not interested in fluctuations in the measured multiplication (whose mean value diverges) but rather in fluctuations in the evolving current. From the time evolution of the mean of the stochastic current and of its standard deviation we can evaluate both the mean time to decision threshold, which limits the communications bit rate, and the standard deviation in this time, also known as the 'jitter'. This latter becomes the more important criterion when communications bandwidth is less important than precision in mean time to decision threshold, such as in laser range finding or in time resolved single photon spectroscopy. These considerations are discussed in the following. 
Numerical simulations ${ }^{82}$ using the RPL technique show that both the mean time to decision threshold and the timing jitter decrease with increasing breakdown probability as the bias is increased above breakdown. They also decrease with decreasing avalanche region width and increasing importance of ionization dead space. Saleh et al. ${ }^{17,83}$ and Groves et al. ${ }^{71}$ showed that Malthusian behaviour also develops above breakdown. Indeed, after an initial, brief transient the mean avalanche current grows exponentially in time by several orders of magnitude before decision threshold is reached. This Malthusian behaviour thus dominates current evolution above breakdown. Groves et al. ${ }^{71}$ further showed that above breakdown, where the Malthusian parameter, $\gamma$ is negative, the standard deviation in current grows exponentially at the same rate, $\sim \exp (-\varkappa)$ as the mean avalanche current. This stands in curious contrast with the behaviour below breakdown ${ }^{17}$, where $\gamma$ is positive, since there it decays exponentially at one half of the rate, $\sim \exp (-\gamma / 2)$ of the mean current. It follows that in this model the jitter becomes independent of time ${ }^{71}$ in this Malthusian regime and this result is confirmed by numerical simulations.

All of these arguments assume very simple models for the devices which are hardly justified in practice. Thus, while Groves et al. ${ }^{71}$ allow the electric field to vary in position in 1-D to deduce Malthusian behaviour above threshold, the field is assumed independent of time. Other models ${ }^{10,17,78,79,80,81,82,83}$ assume the field is uniform everywhere within the multiplication region and at all times. In practice APDs and SPADs possess, at best, approximate radial symmetry, so that reliable, detailed modelling of real devices requires a Monte-Carlo or Fokker-Planck simulator in 2-D. More importantly, in a SPAD the exponentially growing current and associated space charge distort the electric field profile, which then changes in time as well, in turn affecting the evolution of the avalanche. Using detailed numerical studies Spinelli and Lacaita ${ }^{84}$ argue that the lateral spread of avalanche charge triggered at a single point and the resulting spread in times to reach the lateral boundary of the SPAD dominates the jitter.

\section{Conclusions}

The study of impact ionization and avalanche multiplication has been dominated since the 1960 s by the assumptions of the local model, namely that charge carriers can be 
assumed to achieve equilibrium instantaneously with the local electric field in their ability to impact ionise, at least compared with other length and time scales in an experiment, and that they travel always at some saturated drift velocity. The ability of a group of charge carriers to initiate impact ionization is then determined by this local field and not by their spatial or temporal history. This simplifying assumption has informed the interpretation of measurements on avalanche multiplication and noise and the design of APDs for use both below breakdown as noisy, linear amplifiers and above breakdown as single photon detectors. While this local assumption may have been adequate in early Si APDs and SPADs, whose length scale appears to be determined by the small optical absorption coefficient in the indirect band gap, it is not justified in devices comprised of direct gap semiconductors, which can be made much smaller.

Fortunately, while nonlocal multiplication is more difficult to model, it does bring advantages, of reductions in noise, temperature dependence and operating voltage, and of increased speed, so that thin avalanche regions can bring positive benefits, contrary to the predictions of the local model. The interpretation of measurements and the modelling of stochastic processes in this regime have provided stimulating problems with fruitful solutions.

\section{Acknowledgements}

The authors wish to acknowledge EPSRC, EU, DERA Malvern, Agilent, MarconiBookham and DTC for financial support. They also wish to thank the research fellows and students whose hard work and insights have helped us to arrive at our present understanding of these processes and to acknowledge gratefully the specialised help of Richard Tozer and of Peter Robson, who originally invited us down this path and to whose memory this article is dedicated. Chris Groves and Majeed Hayat are thanked for critical readings of the manuscript. 
Figure 1: $\alpha$ measured in $A l_{0.6} G a_{0.4} A$ s pin diodes, with $w=0.027 \mu m(\nabla), 0.0466 \mu m$ $(\Delta), 0.089 \mu \mathrm{m}(\square), 0.512 \mu \mathrm{m}(0)$ and $0.8 \mu \mathrm{m}(\diamond) v s$ inverse electric field. The full line (一) denotes the envelope. SA Plimmer is thanked for the measurements shown in this figure.

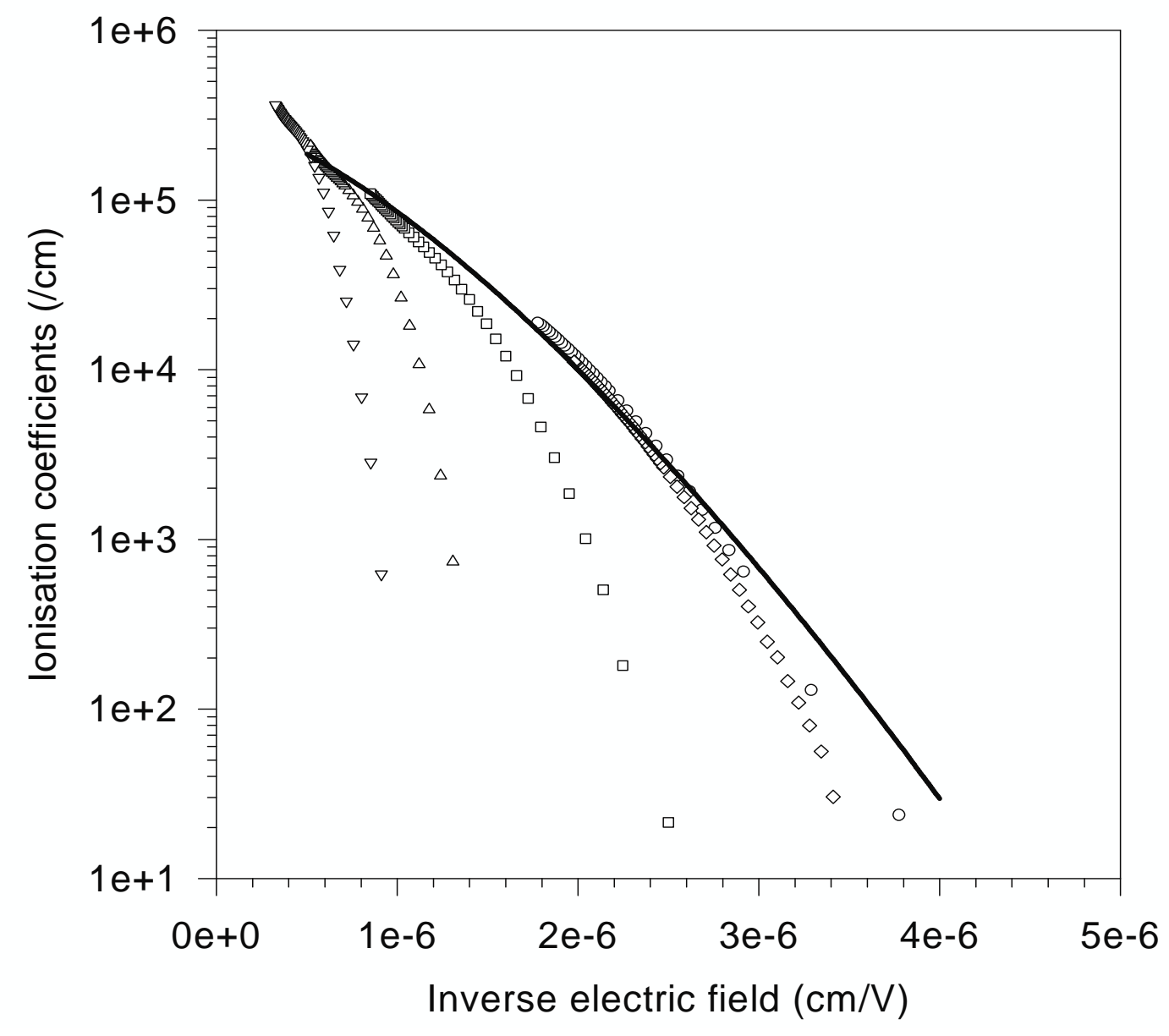


Figure 2: Ionization path length pdfs for electrons (dotted) and holes (full) in GaAs calculated using Monte Carlo. DS Ong is thanked for the calculations shown in this figure

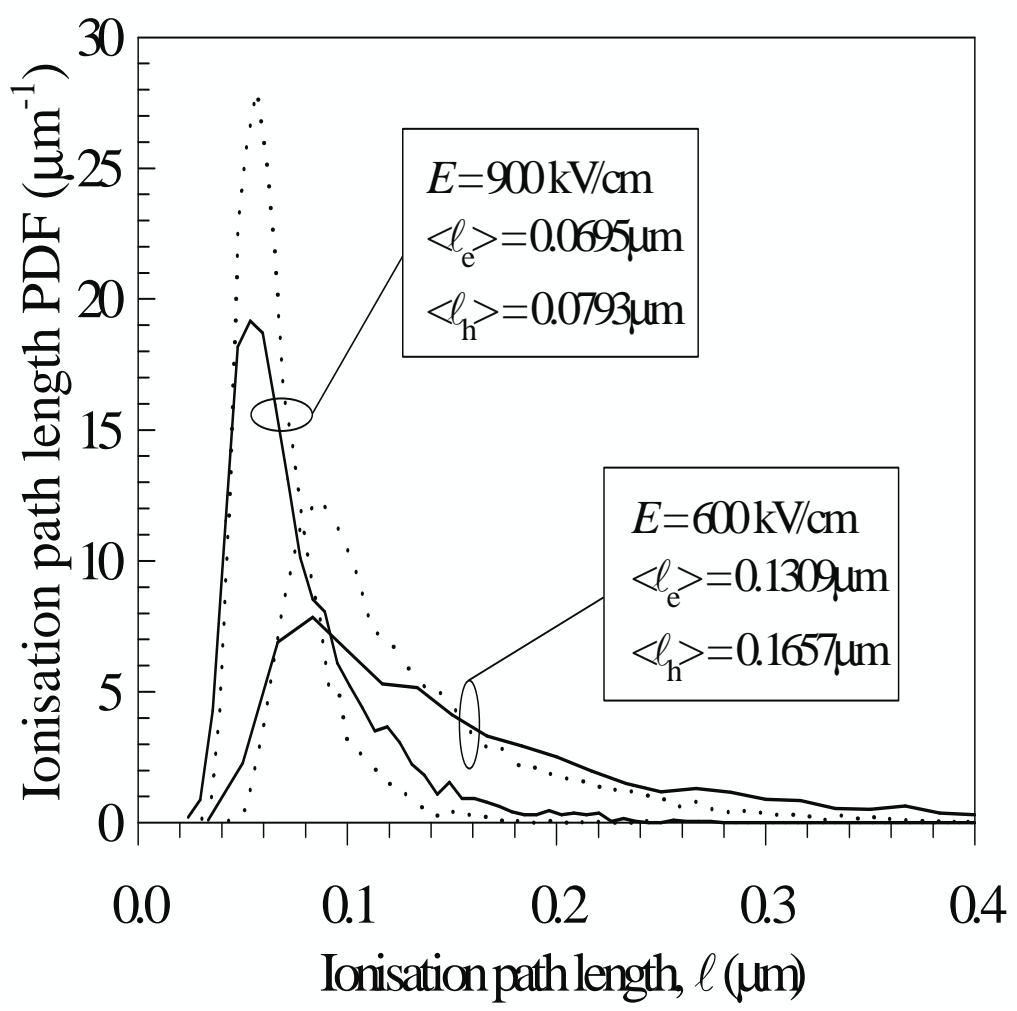


Figure 3: Probability distribution of multiplication $P(M)$ calculated $^{18}$ using Monte Carlo for a $w=0.5 \mu m$ GaAs pin diode, $\langle M\rangle \sim 5$.

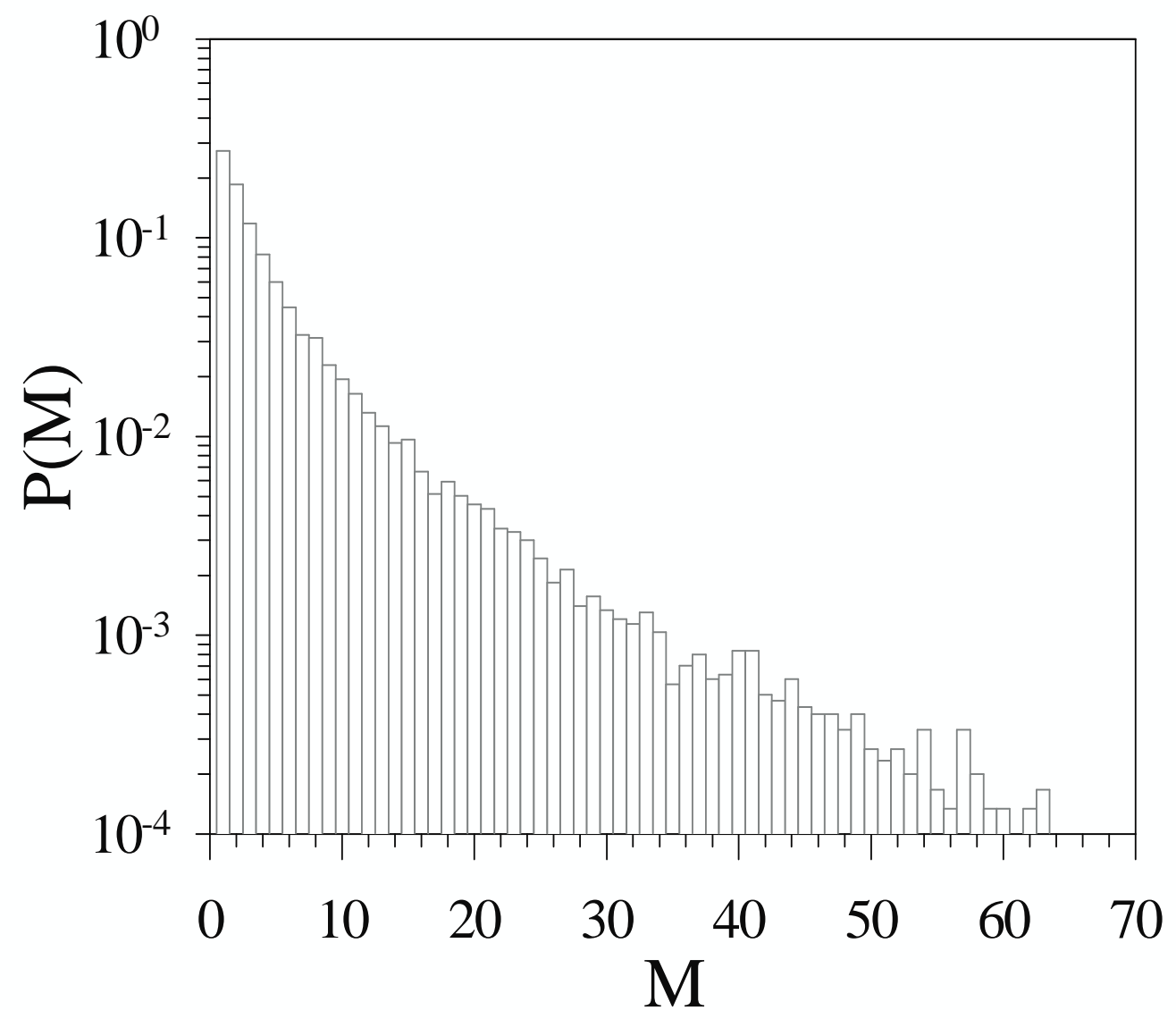


Figure 4: Circuit for measuring avalanche gain and noise using phase sensitive detection $^{21}$.

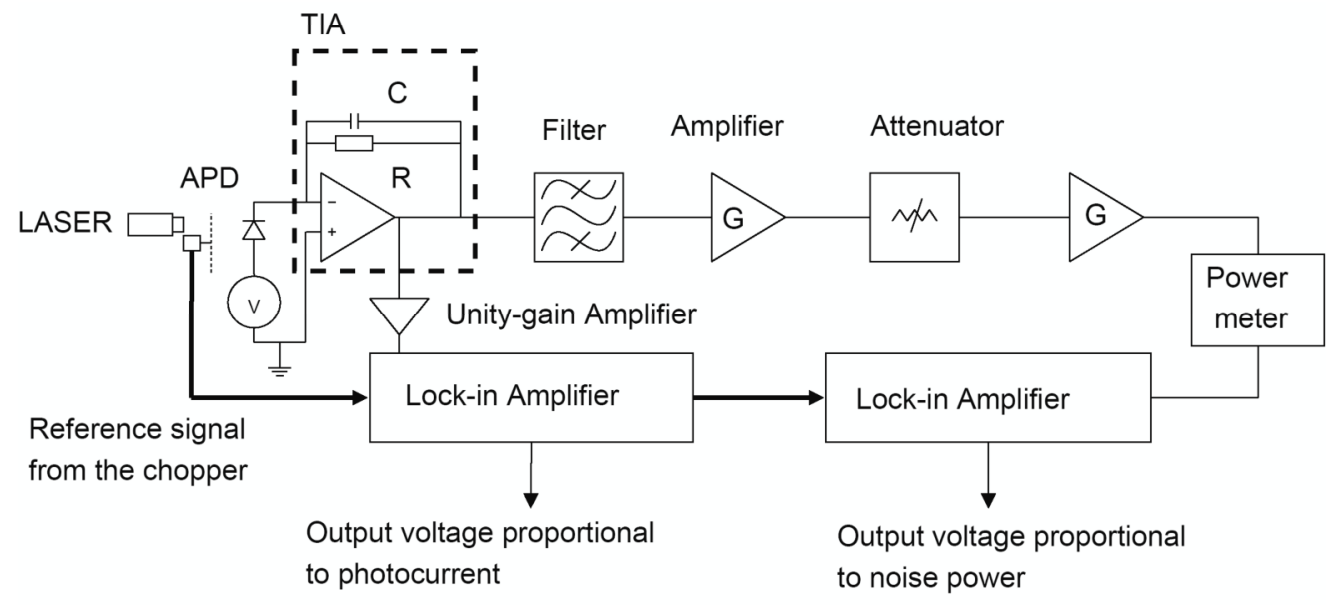


Figure 5: Excess avalanche noise ${ }^{22}$ vs mean multiplication in GaAs pin and nip diodes for values of $w$ ranging from $1.13 \mu \mathrm{m}$ to $0.049 \mu \mathrm{m}$ (pins) and $0.36 \mu \mathrm{m}$ to $0.05 \mu \mathrm{m}$ (nips). Full lines are from eqns. (13) with $k$ increasing from 0 to 1 in steps of 0.1
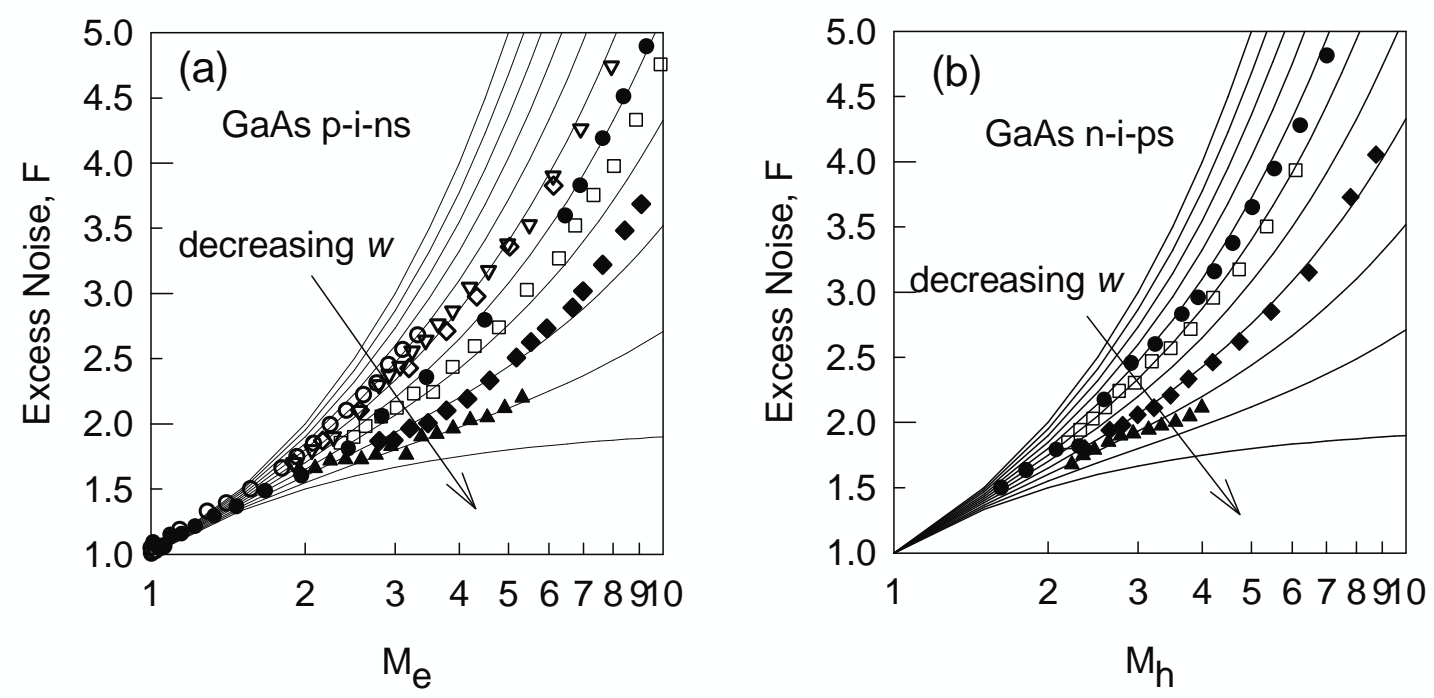
Figure 6: Excess noise ${ }^{32} F$ vs. mean multiplication, $\langle M>$ for pure electron (open) and mixed injection (closed symbols) measured in Si pin diodes, with $w=0.10 \mu m(0,0)$, $0.15 \mu m(\square, \square)$ and $0.29 \mu m(\Delta, \sigma)$. Thin dashed lines are calculated from the local model, while the solid lines (mixed) and thick dashed lines (pure electron injection) are calculated from the nonlocal model.

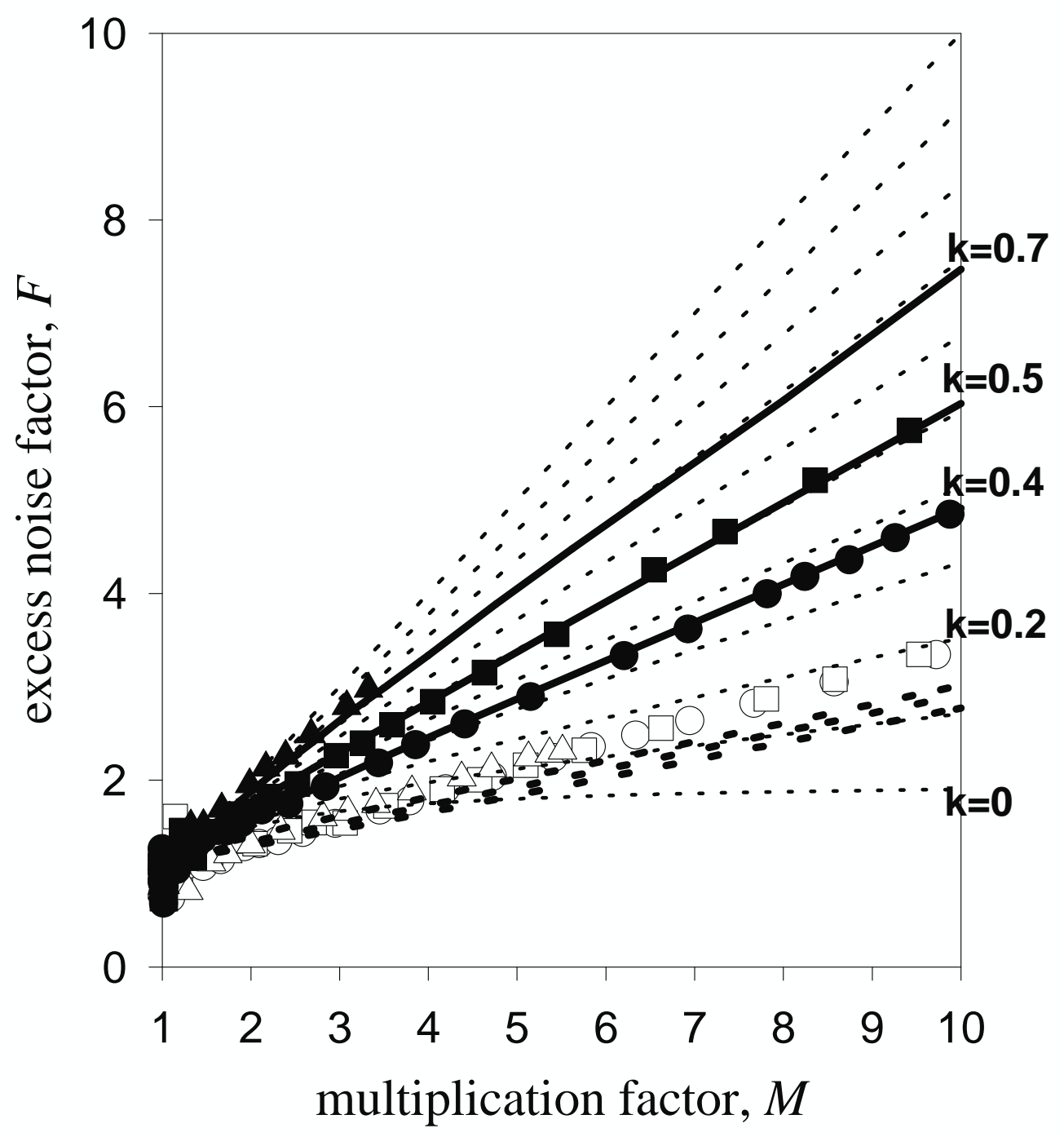


Figure 7: Excess noise vs mean multiplication measured ${ }^{36}$ in $A l_{0.8} G a_{0.2} A$ s pin diodes for pure electron injection as $w$ is decreased from $1.024 \mu \mathrm{m}$ (rectangles) to $0.02 \mu \mathrm{m}$ (hexagons).

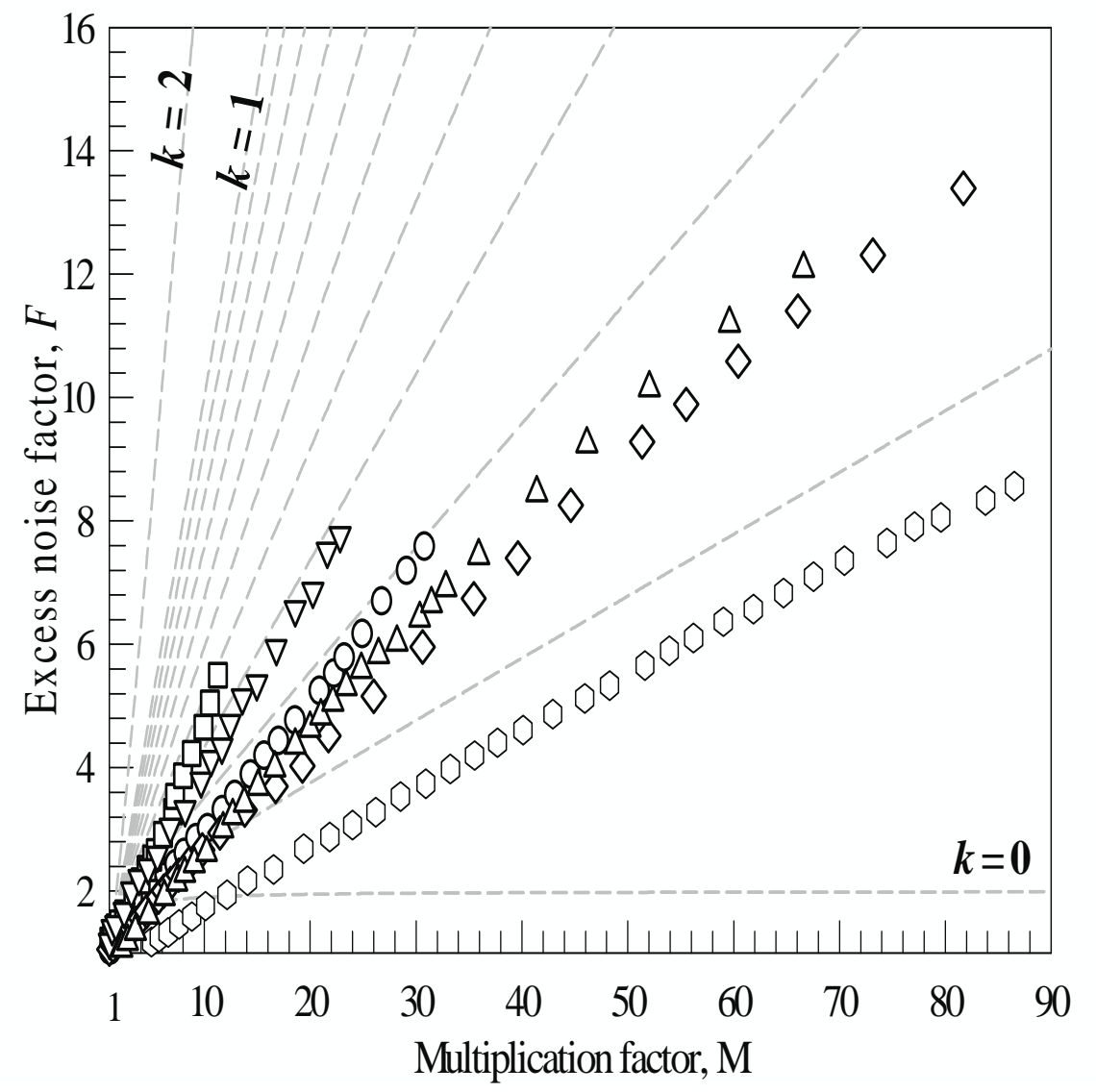


Figure 8: Excess noise characteristics of 4H-SiC pin APDs with a) $w=0.105 \mu m$ and b) $w=0.285 \mu \mathrm{m}$ measured using 365nm (circles) and 230nm (squares) light. Dashed lines are McIntyre's local predictions. B.K. $\mathrm{Ng}$ is thanked for these measurements.
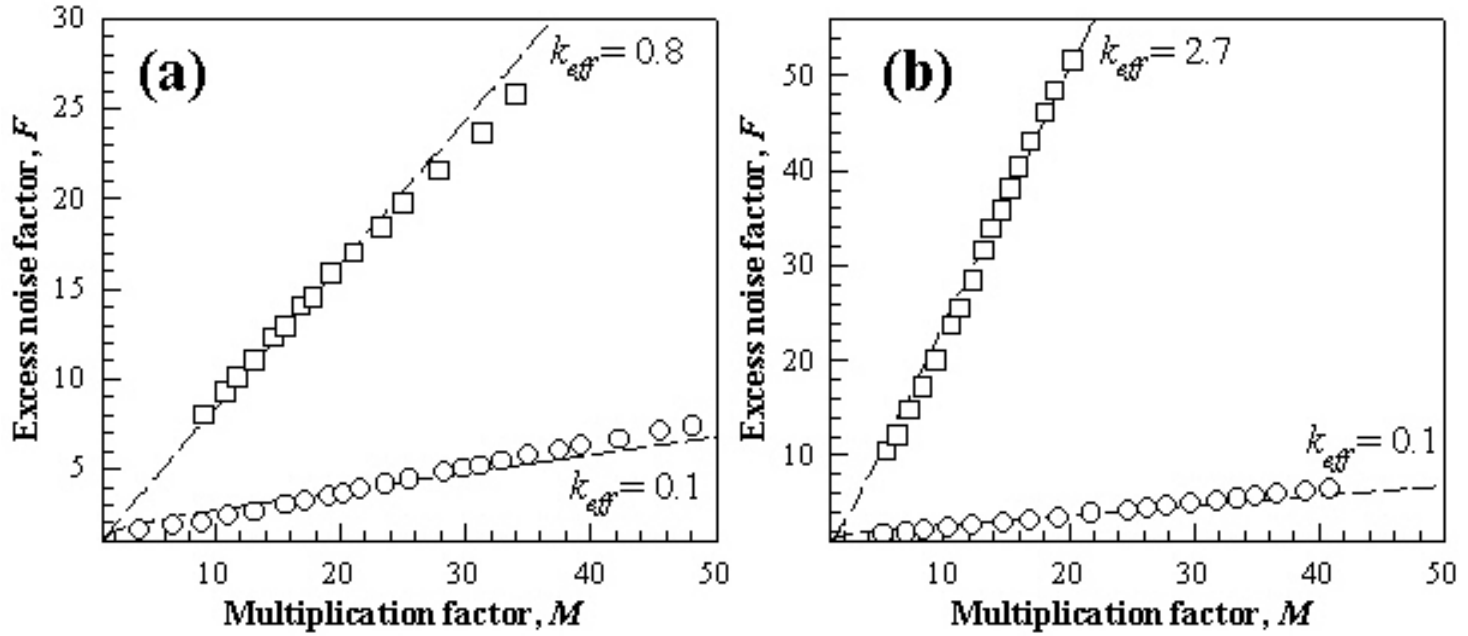
Figure 9: Ionization path length pdfs calculated ${ }^{51}$ from Monte Carlo, Lucky Drift and Fokker-Planck at a field of $500 \mathrm{kV} / \mathrm{cm}$.

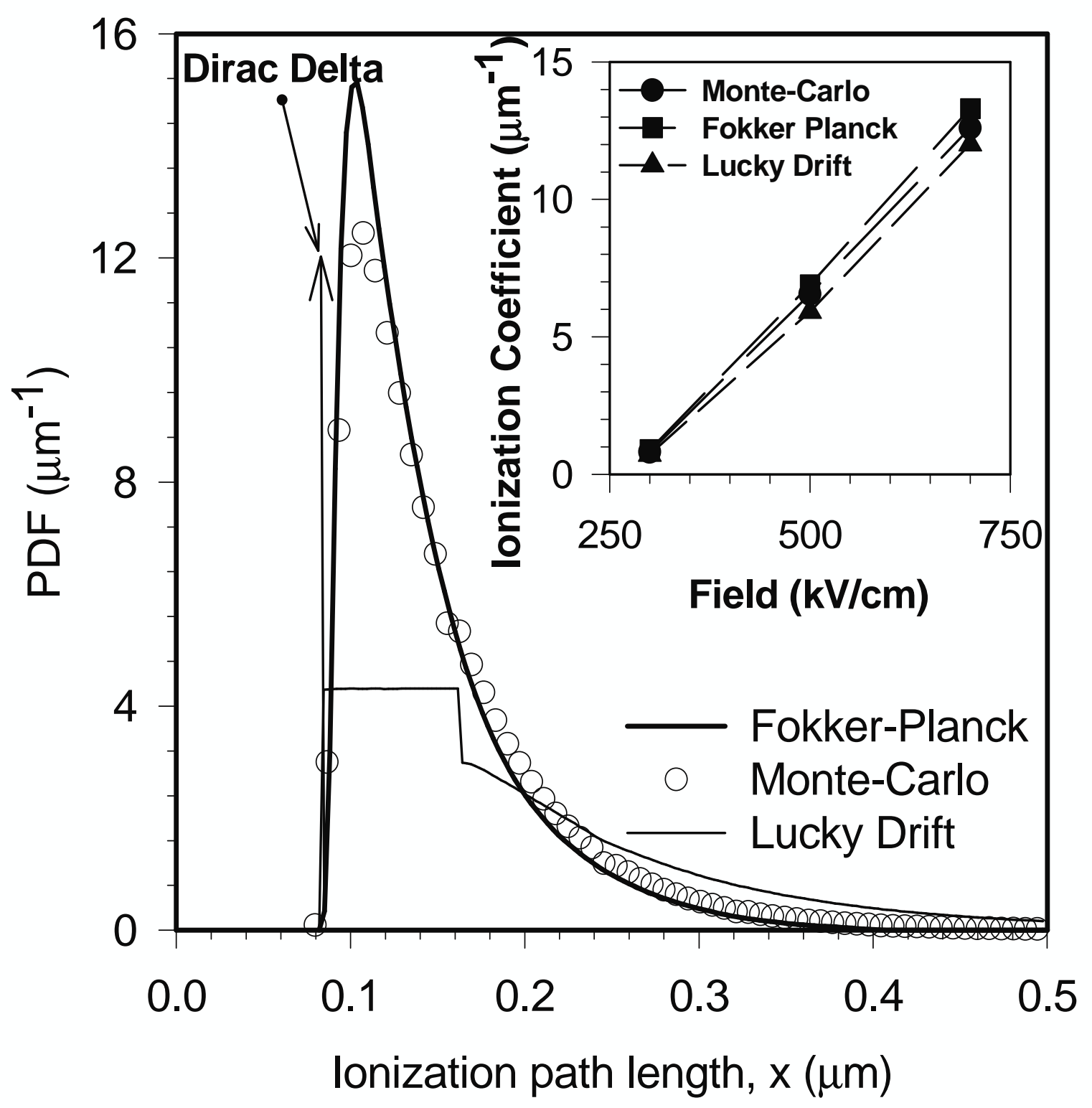


Figure 10: Ionization event pdf, $h(x, t)$ calculated $^{53}$ by Fokker-Planck at $500 \mathrm{kV} / \mathrm{cm}$.

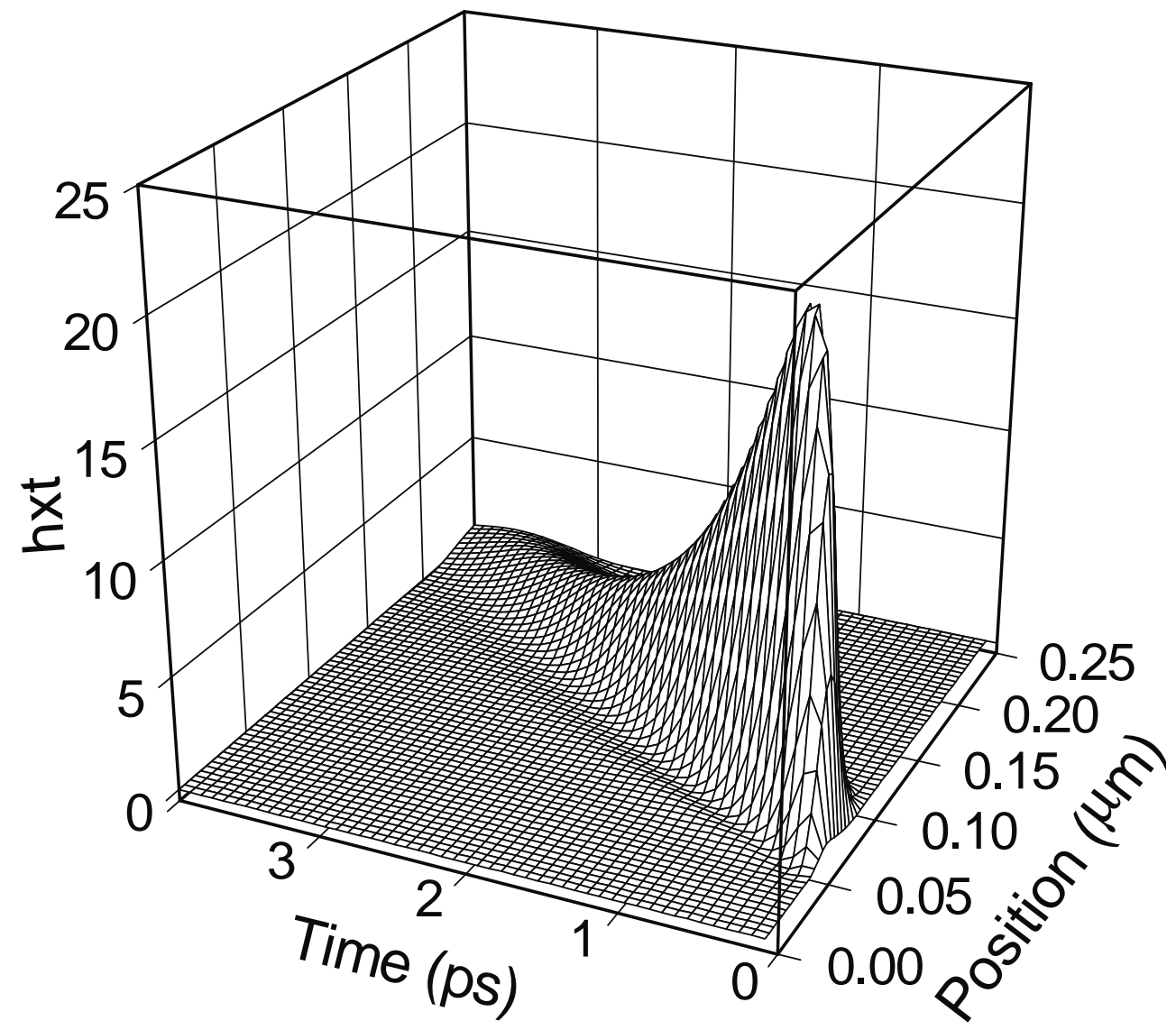


Figure 11: Contour plot of ionization event pdf in space and time, $h(\zeta, \tau)$, generated $^{61}$ by a Simple Monte Carlo model for GaAs at an electric field of $496 \mathrm{kV} / \mathrm{cm}$.

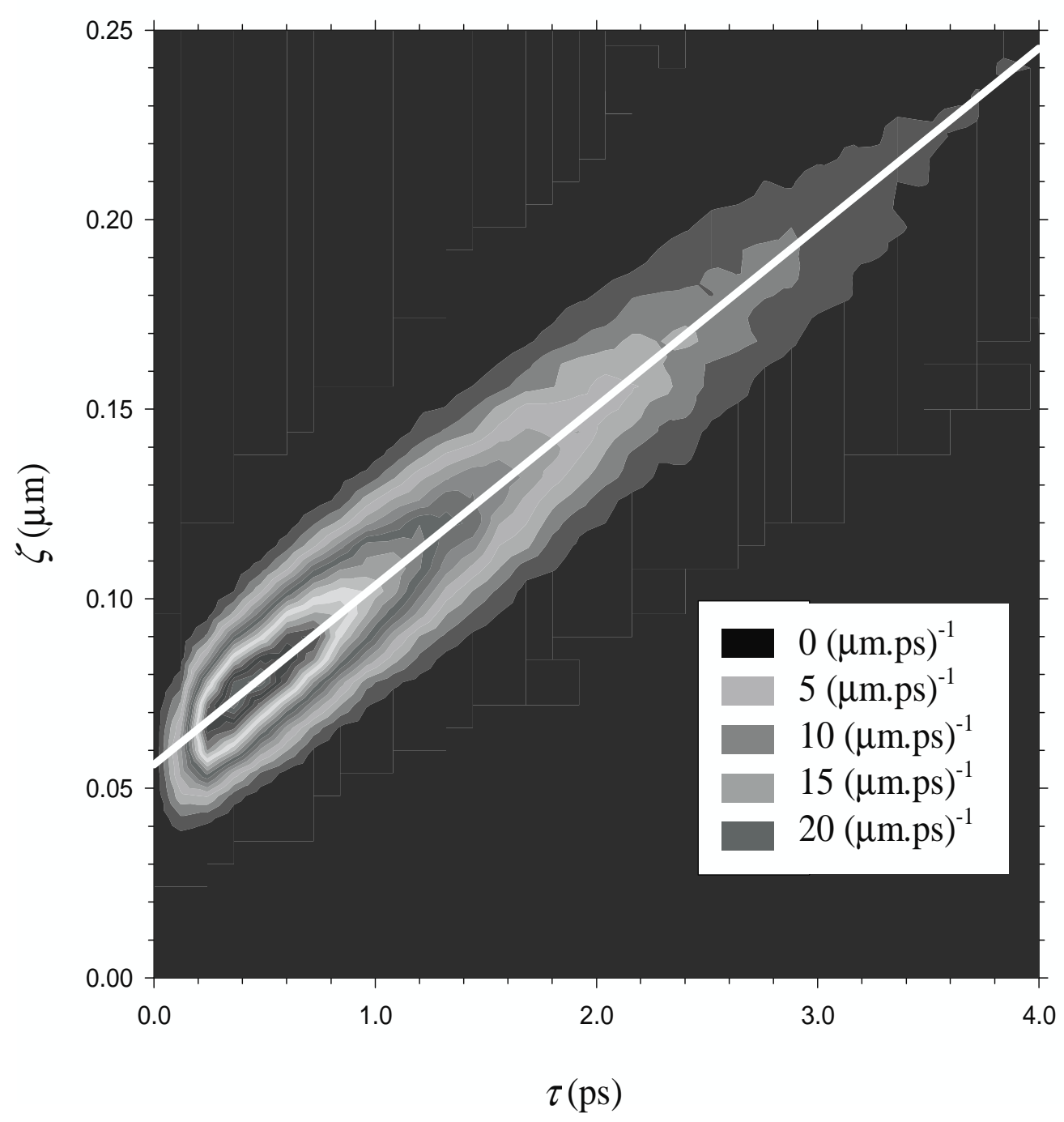


Figure 12: Scaled mean current impulse response, calculated $^{63}$ for diffusion coefficients of $D=0.1 \mathrm{~cm}^{2} \mathrm{~s}^{-1}$ (solid line), $0.2 \mathrm{~cm}^{2} \mathrm{~s}^{-1}$ (dashed line), $1.0 \mathrm{~cm}^{2} \mathrm{~s}^{-1}$ (dash-dotdot-dashed line) and $2.0 \mathrm{~cm}^{2} \mathrm{~s}^{-1}$ (dotted line) for (a) $w=1.0 \mu \mathrm{m}(E=307 \mathrm{kV} / \mathrm{cm})$ and (b) $w=0.2 \mu m(E=496 \mathrm{kV} / \mathrm{cm})$. Also shown are results calculated assuming a constant carrier speed.
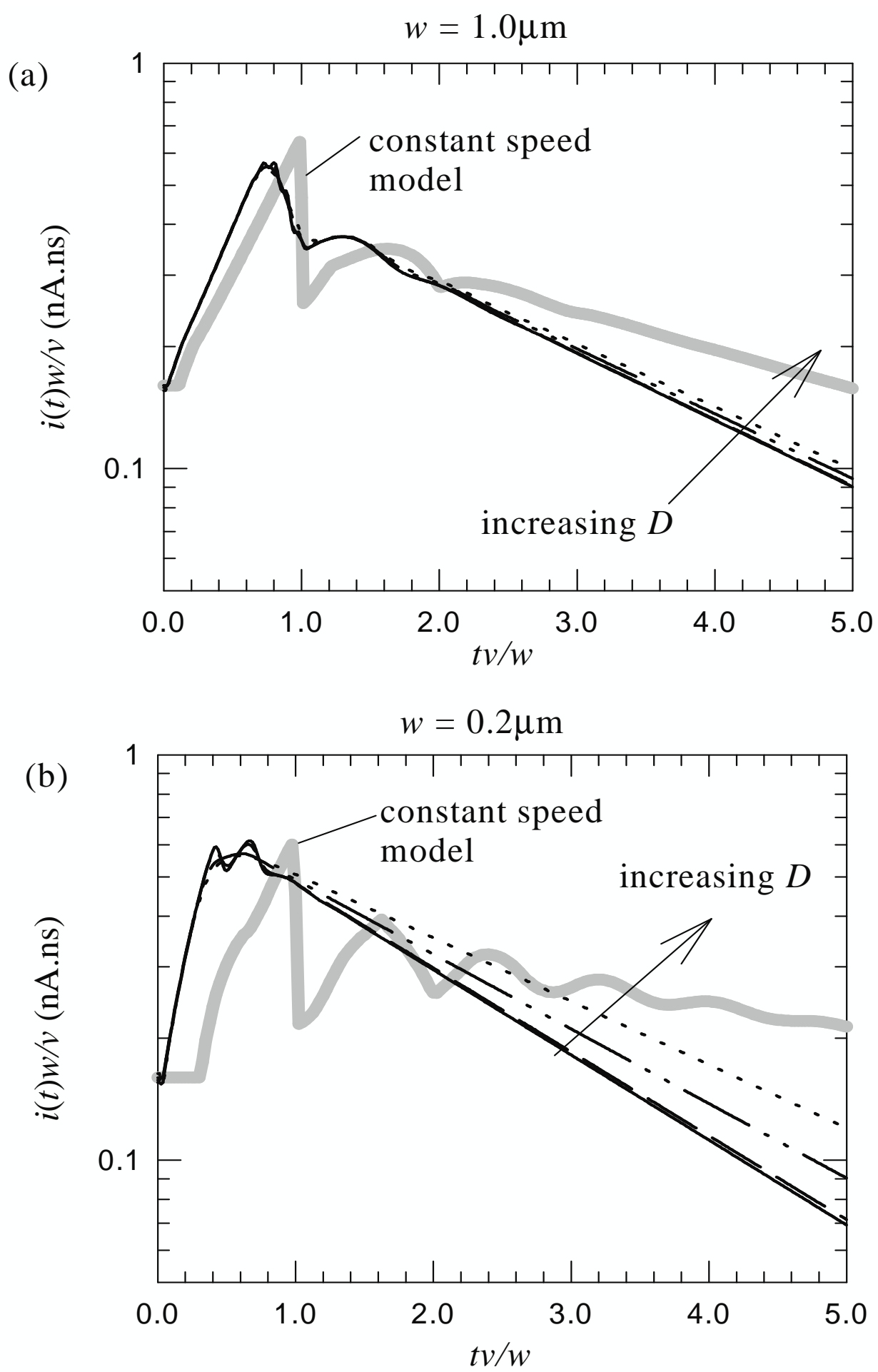
Figure 13: Temperature dependence of multiplication ${ }^{74}$ in $A l_{0.6} G a_{0.4} A s$ in the range $13 \mathrm{~K}$ to $450 \mathrm{~K}$ for $(\mathrm{a}) \mathrm{w}=0.046 \mu \mathrm{m}$ and $0.089 \mu \mathrm{m}$ devices and $(\mathrm{b}) \mathrm{a} w=0.85 \mu \mathrm{m}$ device.
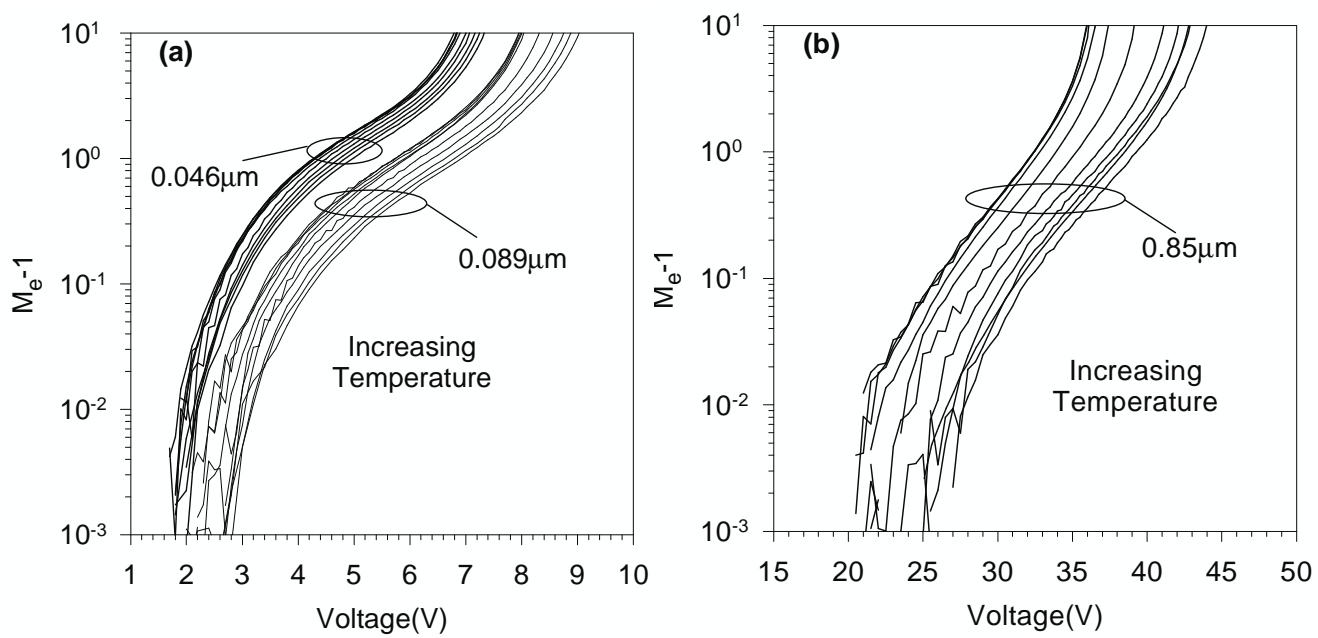


\section{References}

${ }^{1}$ C. Zener, 'A theory of electrical breakdown of solid dielectrics', Proc. Roy. Soc. 145A, $523-9,(1934)$

${ }^{2}$ B. K. Ng, F. Yan, J. P. R. David, R. C. Tozer, G. J. Rees, C. Qin and J. H. Zhao, 'Multiplication and excess noise characteristics of thin $4 \mathrm{H}-\mathrm{SiC} \mathrm{UV}$ avalanche photodiodes', IEEE Photonics Technol. Lett., 14, 1342 - 4 (2002)

${ }^{3}$ D.C. Herbert, C. J. Williams and M. Jaros, 'Impact ionization and noise in SiGe multiquantum well structures', Electron. Letts., 32, 1616 - 8 (1996)

${ }^{4}$ P. Yuan, S. Wang, X. Sun, X.G. Zheng, A.L. Holmes and J.C. Campbell, 'Avalanche photodiodes with an impact ionisation engineered multiplication region', IEEE Photonics Tech. Letts., 12, 1370 - 2 (2000)

${ }^{5}$ J.S. Townsend, 'The genesis of ions by the motion of positive ions in a gas and a theory of the sparking potential'. Phil. Mag. 6, ser. VI, 598 - 618 (1903)

${ }^{6}$ G.E. Stillman and C. M. Wolfe, 'Avalanche photodiodes', Semiconductors and Semimetals (Eds. R. K. Willardson and A. C. Beer, New York Academic Press), 12, 291 - 392 (1977)

${ }^{7}$ F. Capasso, 'Physics of Avalanche Photodiodes', Semiconductors and Semimetals (Eds. R. K. Willardson and A. C. Beer, New York Academic Press), 22, 2 - 172 (1985)

${ }^{8}$ R.J. McIntyre, 'Multiplication noise in uniform avalanche diodes', IEEE Trans. Electron Devices, 13, 164 - 8 (1966)

${ }^{9}$ S.A. Plimmer, J.P.R. David, G.J. Rees and P.N. Robson, 'Ionisation coefficients in $\mathrm{Al}_{\mathrm{x}} \mathrm{Ga}_{1-\mathrm{x}} \mathrm{As}(\mathrm{x}=0$ - 0.6)', Semicond. Sci. Technol., 15692 -9 (2000)

${ }^{10}$ R. J. McIntyre, 'A new look at impact ionization - part 1: a theory of gain, noise, breakdown probability and frequency response', IEEE Trans. Electron Devices, 46, 1623 - 31 (1999)

${ }^{11}$ C.L. Anderson and C.R. Crowell, 'Threshold energies for electron-hole pair production by impact ionization in semiconductors', Phys. Rev. B5 2267-72, (1972)

${ }^{12}$ R. Ghin, J.P.R. David, S.A. Plimmer, M. Hopkinson, G.J. Rees, D.C. Herbert and D.R Wight, 'Avalanche multiplication and breakdown in $\mathrm{Ga}_{0.52} \mathrm{In}_{0.48} \mathrm{P}$ diodes', IEEE Trans. Electron. Dev., 45, 2096 - 101 (1998) 
${ }^{13}$ Y.L. Goh, D.J. Massey, A.R. J. Marshall, J.S. Ng, C.H. Tan, W.K. Ng, G.J. Rees, M. Hopkinson, J.P.R. David and S.K. Jones, 'Avalanche multiplication in InAlAs' IEEE Trans. Electron. Dev., 54, 11 - 16 (2007)

${ }^{14}$ L.J.J. Tan, J.S. Ng, C.H. Tan, M. Hopkinson and J.P.R. David, 'Effect of dead space on low field avalanche multiplication in InP', IEEE Trans. Electron. Dev., 54, $2051-4(2007)$

${ }^{15}$ D.J. Massey, J.P.R. David, C.H. Tan, B.K. Ng, G.J Rees, D.J. Robbins and D.C. Herbert, 'Impact ionisation in submicron Si devices', J. Appl. Phys., 95, 5931 - 3 (2004)

${ }^{16}$ Y. Okuto and C.R. Crowell, 'Ionisation coefficients in semiconductors: a nonlocalised property' Phys. Rev. B 10, 4284 - 96 (1974)

${ }^{17}$ M.M. Hayat, B.E.A. Saleh and M.C. Teich, 'Effect of dead space on gain and noise of double carrier multiplication avalanche photodiodes', IEEE Trans. Electron. Dev., 39, $546-52(1992)$

${ }^{18}$ D.S. Ong, K.F. Li, G.J. Rees, G.M. Dunn, J.P.R. David and P.N. Robson, 'A Monte Carlo investigation of multiplication noise in thin $\mathrm{p}^{+}$in $^{+} \mathrm{GaAs}$ avalanche photodiodes' IEEE Trans. Electron. Dev. 45, 1804 - 10 (1998)

${ }^{19}$ e.g. A. Van der Ziel, ‘Solid State Physical Electronics’ Prentice Hall (1965)

${ }^{20}$ S.Ramo, 'Currents induced by electron motion', Proceedings of the I.R.E, 584-585, September (1939)

${ }^{21}$ K.S. Lau, C.H. Tan, B.K. Ng, K.F. Li, R.C. Tozer, J.P.R. David and G.J. Rees, 'Excess noise measurement in avalanche photodiodes using a transimpedance amplifier front end', Meas. Sci. Technol. 17, 1941 - 6 (2006)

${ }^{22}$ K.F. Li, D.S. Ong, J.P.R. David, G.J. Rees, R.C. Tozer, P.N. Robson and R. Grey,'Avalanche multiplication noise characteristics in thin $\mathrm{GaAs} \mathrm{p}^{+}$in $^{+}$diodes', IEEE Trans. Electron Dev., 45, 2102 - 7 (1998)

${ }^{23}$ C. Hu, K.A. Anselm, B.G. Streetman and J.C. Campbell, 'Noise characteristics of thin multiplication region GaAs avalanche photodiodes', 'Appl. Phys. Letts.', 69, $3734-6,(1996)$

${ }^{24}$ D.C. Herbert, 'Avalanche noise in submicron pin diodes', Electron. Letts., 33, 1257 $-8(1997)$

${ }^{25}$ K.F. Li, D.S. Ong, J.P.R.David, R.C. Tozer, G.J. Rees, P.N. Robson and R. Grey, 'Low excess noise characteristics in thin avalanche region GaAs diodes' Electron. Letts. 34, 125 - 6 (1998) 
${ }^{26}$ K.F. Li, D.S. Ong, J.P.R. David, R.C. Tozer, G.J. Rees, P.N. Robson and R. Grey, 'Low noise GaAs and $\mathrm{Al}_{0.3} \mathrm{Ga}_{0.7} \mathrm{As}$ avalanche photodetectors' IEEE Proc.

Optoelectron. 146, $21-4$ (1999)

${ }^{27}$ X.G. Zheng, X. Sun, S. Wang, P. Yuan, G.S. Kinsey, A.L. Holmes, B.G. Streetman and J.C. Campbell, 'Multiplication noise of $\mathrm{Al}_{\mathrm{x}} \mathrm{Ga}_{1-\mathrm{x}} \mathrm{As}$ avalanche photodiodes with high Al concentration and thin multiplication region', Appl. Phys. Letts. 78, 3833 - 5 (2001)

${ }^{28}$ C.H. Tan, J.P.R. David, S.A. Plimmer, G.J. Rees, R.C. Tozer and R. Grey, 'Low multiplication noise thin $\mathrm{Al}_{0.6} \mathrm{Ga}_{0.4}$ As avalanche photodiodes', IEEE Trans Electron Dev. 48, 1310 - 7 (2001)

${ }^{29}$ C.H. Tan, R. Ghin, J.P.R. David, G.J. Rees and M. Hopkinson, 'The effect of dead space on gain and excess noise in $\operatorname{In}_{0.48} \mathrm{Ga}_{0.52} \mathrm{P} \mathrm{p}^{+}$in $^{+}$diodes', Semicond. Sci. Technol. 18, $803-6(2003)$

${ }^{30}$ P. Yuan, C.C. Hansing, K. Anselm, C.V. Lennox, H. Nie, A.L. Holmes, B.G. Sreetman and J.C. Campbell, 'Impact ionisation characteristics of III-V semiconductors for a wide range of multiplication thicknesses', IEEE JQE, 36, 198 204 (2000)

${ }^{31}$ Y.L. Goh, A.R.J. Marshall, D.J. Massey, J.S. Ng, C.H. Tan, M. Hopkinson, J.P.R. David, S,K, Jones, C.C. Button and S.M. Pinches, 'Excess avalanche noise in $\mathrm{In}_{0.52} \mathrm{Al}_{0.48} \mathrm{As}$ ', IEEE JQE 43, 503 - 7 (2007)

${ }^{32}$ C.H. Tan, J.C. Clark, J.P.R. David, G.J. Rees, S.A. Plimmer, R.C. Tozer, D.C. Herbert, D.J. Robbins, W.Y. Leong and J. Newey, 'Avalanche noise measurement in thin Si p ${ }^{+}{ }^{+}{ }^{+}$diodes' Appl. Phys Letts., 76, 3926 - 8 (2000)

${ }^{33}$ K.M. Van Vliet, A. Friedmann and L.M. Rucker, 'Theory of carrier multiplication and noise in avalanche devices part II: two carrier processes

${ }^{34}$ B.K. Ng, J.P.R. David, S.A. Plimmer, M. Hopkinson, R.C. Tozer and G.J. Rees, 'Impact ionisation coefficients of $\mathrm{Al}_{0.8} \mathrm{Ga}_{0.3} \mathrm{As}$ ' Appl. Phys. Lett., 77, 4374 - 6 (2000)

${ }^{35}$ B.K. Ng, J.P.R. David, G.J. Rees, R.C. Tozer, M. Hopkinson and R.J. Airey, 'Avalanche multiplication and breakdown in $\mathrm{Al}_{\mathrm{x}} \mathrm{Ga}_{1-\mathrm{x}} \mathrm{As}(\mathrm{x}<0.9)$ ', IEEE Trans. Electron Dev. 49, 2349 - 51 (2002)

${ }^{36}$ B. K. Ng, J. P. R. David, R. C. Tozer, M. Hopkinson, G. Hill and G. J. Rees, 'Excess noise characteristics of $\mathrm{Al}_{0.8} \mathrm{Ga}_{0.2} \mathrm{As}$ avalanche photodiodes' IEEE Photonics Tech. Letts., 14, 522 - 4 (2002)

${ }^{37}$ B. K. Ng, F. Yan, J. P. R. David, R. C. Tozer, G. J. Rees, C. Qin and J. H. Zhao, 'Multiplication and excess noise characteristics of thin 4H-SiC UV avalanche photodiodes', IEEE Photonics Technol. Lett., 14, 1342 - 4 (2002) 
${ }^{38}$ B. K. Ng, J. P. R. David, R. C. Tozer, G. J. Rees, F. Yan, J. H. Zhao, and M. Weiner, 'Nonlocal effects in thin $4 \mathrm{H}-\mathrm{SiC}$ UV avalanche photodiodes' IEEE Trans. Electron Dev. 50, 1724 - 32 (2003)

${ }^{39}$ F. Ma, X. Li, J.C. Campbell, J.D. Beck, C.-F. Wan and M.A. Kinch, 'Monte Carlo simulations of $\mathrm{Hg}_{0.7} \mathrm{Cd}_{0.3} \mathrm{Te}$ avalanche photodiodes and resonance phenomena in the multiplication noise' Appl. Phys. Lett., 83785 - 7 (2003)

${ }^{40}$ M.A. Kinch, J.D. Beck, C.-F. Wan, F. Ma and J.C. Campbell, 'HgCdTe electron avalanche photodiodes' J. Electron. Mats., 33, 630 - 9 (2004)

${ }^{41}$ D.C. Herbert, P.A. Childs, R.A. Abram, G.C. Crow and M. Walmsley, 'Selfconsistent 2-D Monte Carlo simulations of InSb APD', IEEE Trans Electron. Dev., 52, $2175-81(2005)$

${ }^{42}$ A.R.J. Marshall, C.H. Tan, M.J. Steer and J.P.R. David, 'Electron dominated impact ionisation and avalanche gain characteristics in InAs photodiodes', Appl. Phys. Lett., 93, 111107-9 (2008)

${ }^{43}$ A.R.J. Marshall, C.H. Tan, M.J. Steer and J.P.R. David, 'Extremely low excess noise in InAs electron avalanche photodiodes' IEEE Photonics Tech. Letts., 21, 866 8 (2009)

${ }^{44}$ M.M. Hayat, W.L. Sargeant and B.A. Saleh, 'Effect of dead space on gain and noise in Si and GaAs avalanche photodiodes', IEEE J. Quantum Electron., 28, 1360 5 (1992)

${ }^{45}$ M.M. Hayat, O.-H. Kwon, S. Wang, J.C. Campbell, B.E.A. Saleh and M.C. Teich, 'Boundary effects on multiplication noise in thin heterostructure avalanche photodiodes: theory and experiment' IEEE Trans. Electron Dev., 49, 2114 - 23 (2002)

${ }^{46}$ C. Jacoboni and M. Reggiani, 'The Monte Carlo method for the solution of charge transport in semiconductors with applications to covalent materials', Rev. Mod. Phys. $\mathbf{5 5}, 645-705$ (1983)

${ }^{47}$ B.K. Ridley, 'The lucky-drift mechanism of impact ionization', J. Phys. C, 16, 3373 - 88 (1983)

${ }^{48}$ M.G. Burt, 'An alternative expression for the impact ionization coefficient in a semiconductor derived using the lucky drift theory', J. Phys. C., 18, L477 - 81 (1985)

${ }^{49}$ S. McKenzie and M.G. Burt, 'A test of the lucky drift theory of the impact ionization coefficient using Monte Carlo simulation', J. Phys. C., 18, 1959 - 73 (1986)

${ }^{50}$ B. Jacob, S. A. Plimmer, P. N. Robson and G. J. Rees, 'Lucky drift model for nonlocal impact ionization', IEE Proc. Optoelectron., 148, 81 -3 (2001) 
${ }^{51}$ B. Jacob, P. N. Robson, J. P. R. David and G. J. Rees, 'Fokker-Planck model for nonlocal impact ionization in semiconductors', J. Appl. Phys., 90, 1314 - 7 (2001)

${ }^{52}$ E. Bringuier, 'Augmented Fokker-Planck equation for electron transport in arbitrary fields', J. Appl. Phys., 86, 6847 - 51 (1999)

${ }^{53}$ B. Jacob, P. N. Robson, J. P. R. David and G. J. Rees, 'Fokker-Planck approach to impact ionization distributions in space and time', J. Appl. Phys., 91, $5438-41$ (2002)

${ }^{54}$ S.A.Plimmer, J.P.R.David, D.S.Ong and K.F.Li, 'A simple model for avalanche multiplication including deadspace effects', IEEE Trans. Electron Dev., 46, 769 - 75 (1999)

${ }^{55}$ D.S. Ong, K.F. Li, G.J. Rees, J.P.R. David and P.N. Robson, 'A simple model to determine multiplication and noise in avalanche photodiodes', J. Appl. Phys., 83, $3426-8$ (1998)

${ }^{56}$ R. B. Emmons, 'Avalanche photodiode frequency response', J. Appl. Phys., 38, $3705-14$ (1967)

${ }^{57}$ R. Kuvås and C. A. Lee, 'Quasistatic approximation for semiconductor avalanches', J. Appl. Phys., 41, 1743 - 55 (1970)

${ }^{58}$ B. E. A. Saleh, M. M. Hayat and M. C. Teich, 'Effect of dead space on the excess noise factor and time response of avalanche photodiodes', IEEE Trans. Electron Dev., 37, 1976 - 84 (1990)

${ }^{59}$ M.M. Hayat and B.E.A. Saleh, 'Statistical properties of the impulse response function of double carrier multiplication avalanche photodiodes including the effect of dead space', J. Lightwave Technol., 10, 1415 - 25 (1992)

${ }^{60}$ J. S. Ng, C. H. Tan, B. K. Ng, P. J. Hambleton, J. P. R. David, G. J. Rees, A. H. You and D. S. Ong, 'Effect of dead space on avalanche speed', IEEE Trans. Electron Dev., 49, 544 - 9 (2002)

${ }^{61}$ S. A. Plimmer, J. P. R. David, B. Jacob and G. J. Rees, 'Impact ionization probabilities as functions of two-dimensional space and time', J. Appl. Phys., 89, $2742-51(2001)$

${ }^{62}$ D. S. Ong, G. J. Rees and J. P. R. David, 'Avalanche speed in thin avalanche photodiodes', J. Appl. Phys., 93, 4232-4239 (2003)

${ }^{63}$ C.H. Tan, P.J. Hambleton, J.P.R. David, R.C. Tozer, and G.J. Rees, 'Calculation of APD impulse response using a space- and time-dependent ionization probability distribution function' IEEE J. Lightwave Technol., 21, 155 - 9 (2003)

${ }^{64}$ P. J. Hambleton, S. A. Plimmer and G. J. Rees, 'Limitations of the saturated drift velocity approximation for time domain modelling', Semicond. Sci. Technol., 17, 124 $-8(2002)$ 
${ }^{65}$ P.J. Hambleton, S.A. Plimmer, J.P.R. David and G.J. Rees, 'Time response modelling in submicron avalanche photodiodes' 'IEE Proc. Optoelectron.', 150, 167$70(2003)$

${ }^{66}$ P.J. Hambleton, S.A. Plimmer, J.P.R. David, G.J. Rees and G.M. Dunn, 'Simulated current response in avalanche photodiodes' J. Appl Phys., 91, 2107 - 111 (2002)

${ }^{67}$ M. M. Hayat and G. Dong, 'A new approach for computing the bandwidth statistics of avalanche photodiodes', IEEE Trans. Electron Dev., 47, 1273 - 9 (2000)

${ }^{68}$ P. Sun, M.M. Hayat, B.E.A. Saleh and M.C. Teich, 'Statistical correlation of gain and buildup time in APDs and its effects on receiver performance', J. Lightwave Technol., 24, 755 - 68 (2006)

${ }^{69}$ J. S. Ng, C. H. Tan, J. P. R. David and G. J. Rees, 'A general method for estimating the duration of avalanche multiplication', Semicon. Sci. and Technol., 17, $1067-71$ (2002)

${ }^{70}$ K.B. Athreya and P. Ney., 'Branching processes' Berlin-Germany: Springer-Verlag (1972)

${ }^{71}$ C. Groves, C.H. Tan, J.P.R. David, G.J. Rees and M.M. Hayat, 'Exponential time response in analogue and Geiger mode avalanche photodiodes', IEEE Trans.

Electron. Dev., 52, $1527-34$ (2005)

${ }^{72}$ F. Ma, G. Karve, X. Zheng, X. Sun, A. L. Holmes and J. C. Campbell, 'Low temperature breakdown properties of $\mathrm{Al}_{\mathrm{x}} \mathrm{Ga}_{1-\mathrm{x}} \mathrm{As}$ avalanche photodiodes', Appl. Phys. Lett., 81, 1908 - 10 (2002)

${ }^{73}$ C.N. Harrison, J. P. R. David, M. Hopkinson and G. J. Rees, 'Temperature dependence of avalanche multiplication in submicron $\mathrm{Al}_{0.6} \mathrm{Ga}_{0.4} \mathrm{As}$ diodes' J. Appl. Phys, 92, $7684-6$ (2002)

${ }^{74}$ C.N. Harrison, J.P.R. David, C. Groves, M. Hopkinson and G.J. Rees, 'Effect of temperature on the avalanche properties of sub-micron devices' Inst. Phys. Conf. Ser., 174, section 4, 263 - 6 (2003)

${ }^{75}$ C. Groves, R. Ghin, J.P.R. David and G.J. Rees, 'Temperature Dependence of Impact Ionization in GaAs', IEEE Trans. Electron. Dev., 50, 2027 - 31 (2003)

${ }^{76}$ D.J. Massey, J.P.R. David and G.J. Rees, 'Temperature dependence of impact ionisation in submicron silicon devices', IEEE Trans. Electron Dev., 53, 2328 - 34 (2006)

${ }^{77}$ C. Groves, J.P.R. David and G.J. Rees, 'The effect of ionisation threshold softness on the temperature dependence of the impact ionisation coefficient', Semicond Sci. and Technol., 18, 689 -92 (2003) 
${ }^{78}$ W.G. Oldham, R.R. Samuelson, and P. Antognetti, 'Triggering phenomena in avalanche photodiodes', IEEE Trans. Electron. Dev., 19, 1056 - 60 (1972)

${ }^{79}$ R.J. McIntyre, 'On the avalanche initiation probability of avalanche diodes above the breakdown voltage', IEEE Trans. Electron. Dev., 20, 637 - 41 (1973)

${ }^{80}$ S. Wang, F. Ma, X. Li, G. Karve, X. Zheng and J.C. Campbell 'Analysis of breakdown probabilities in avalanche photodiodes using history-dependent analytical model' Appl. Phys. Lett., 821971 - 3 (2003)

${ }^{81}$ J. S. Ng, C. H. Tan, G. J. Rees and J. P. R. David, 'Effects of dead space on breakdown probability in Geiger mode avalanche photodiode', J. Modern Optics, 54, $353-360(2007)$

${ }^{82}$ C.H.Tan, J.S.Ng, G.J.Rees and J.P.R.David, 'Statistics of avalanche current build up time in single photon avalanche diodes' IEEE J. Selected topics in quantum electronics 13, 906 - 910 (2007)

${ }^{83}$ M.A. Saleh, M.M. Hayat, O.-H. Kwon, A.L. Holmes, J.C. Campbell, B.E. A. Saleh and M.C. Teich, 'Breakdown voltage in thin III-V avalanche photodiodes', Appl. Phys. Letts., 79, 4037 - 9 (2001)

${ }^{84}$ A Spinelli and A.L. Lacaita, 'Physics and numerical simulation of single photon avalanche photodiodes', IEEE Trans. Electron Dev., 44, 1931 - 43 (1997) 